\title{
Die Gegnerlnnenschaft zur Ehe für alle: ein autoritär-regressives politisches Projekt?
}

\author{
CHRISTOPHER FritZSCHE \& JULIANE LANG ${ }^{1}$
}

\section{EINLEITUNG}

Für die einen war es ein unerwarteter Erfolg, die Frucht langer Jahre der politischen Arbeit, für die anderen das ,Ende der Ehe“ oder gar der Beginn vom, Ende der Familie': Die Verabschiedung des Gesetzes zur sogenannten Ehe für alle ${ }^{2}$ im Juni 2017 war für Befürworter_innen und GegnerInnen ${ }^{3}$ ein Moment großer Emotionen - und stellt für uns ein diskursives Ereignis dar, anhand dessen wir das Ringen unterschiedlicher politischer Kräfte um Einfluss auf die sich im Wandel befindlichen Geschlechter- und Sexualitätsverhältnisse betrachten wollen.

In den vergangenen Jahrzehnten ist eine Gleichzeitigkeit geschlechter- und sexualitätsbezogener Liberalisierungen (Lenz 2013) und einer Radikalisierung des Widerstands gegen dieselben zu beobachten. Die Öffnung der Ehe für gleichgeschlechtliche Partnerschaften ist in den vergangenen Jahren nicht nur in Deutschland, sondern auch in vielen anderen Ländern eine der Hauptarenen, in denen die Auseinandersetzungen um Deutungsmacht über legitimes und illegitimes, von den GegnerInnen häufig als ,ideologisch` bezeichnetes Geschlechterwissen geführt wird (Kuhar/Paternotte 2017). Diese Konflikte um die Ehe für alle analysieren wir als

1 Wir danken Annette Henninger und Ursula Birsl für hilfreiche Anmerkungen zum Text. Zudem danken wir Anne Gehrmann und Melanie Kuhnert, die als studentische Hilfskräfte an unserer Fallstudie mitgewirkt haben.

2 Zum Begriff: siehe Kapitel 5.

3 Die GegnerInnen der Ehe für alle definieren sich weltanschaulich gerade über ihr Festhalten an einem heteronormativen Weltbild. Um auf diesen Umstand hinzuweisen, gendern wir in diesem Kontext mit dem Binnen-I (vgl. auch: Sanders/Jentsch/Hansen 2014: 5), während wir ansonsten den Unterstrich verwenden. 
paradigmatisch für die Formierung eines politischen Projekts, dessen Agenda wir als autoritär-regressiv charakterisieren. Denn es sind Themen rund um die Ordnung von Geschlecht und Sexualität, die als Triggerpunkte für unterschiedlichste autoritär-rechte und konservative Akteure fungieren und von diesen untermalt werden mit angsterfüllten Fantasien eines Verlustes jedweder Form eindeutiger Identitäten.

Wir gehen in diesem Beitrag der Forschungsfrage nach, inwieweit sich in der Gegnerschaft zur Ehe für alle ein politisches Projekt formiert. Hierbei stützen wir uns auf die Annahme der Historisch-Materialistischen Politikanalyse, dass solche politischen Projekte Teil von größeren Hegemonieprojekten sein können, mit deren Hilfe Akteursbündnisse längerfristig nach gesellschaftlicher Deutungshoheit streben. Im Kern des hier als autoritär-regressiv bezeichneten politischen Projektes steht die Verteidigung einer Eindeutigkeit gegenüber einer Vieldeutigkeit geschlechtlicher und sexueller Identitäten. Hierbei gilt es, neben den ideologischen immer auch die ökonomischen Bedingungen der Formierung eines solchen politischen Projektes miteinzubeziehen. Um Letzteres aufzuzeigen, greifen wir in unserer Fallstudie auf eine feministisch gewendete Regulationstheorie zurück (Henninger/ Backöfer/Fritzsche/Näser-Lather in diesem Band).

Im Mittelpunkt unserer Analyse steht weniger die Frage nach dem Erfolg dieses politischen Projektes, sondern der Prozess seiner Formierung. Dies gibt uns, so unsere These, Aufschluss über die Anschluss- und Mobilisierungsfähigkeit weiterer politischer Projekte, die um die Verteidigung von Geschlechter- und Sexualitätsverhältnissen in ihrer patriarchalen Form ringen.

Den von uns hier eingeführten Begriff des autoritär-regressiven Projektes erläutern wir gleich eingangs in unserem theoretischen Zugriff. Anschließend kombinieren wir den im Projektzusammenhang entwickelten regulationstheoretischen $\mathrm{Zu}$ gang mit dem hegemonietheoretischen Ansatz der Historisch-Materialistischen Politikanalyse (HMPA). Den Prozess der Formierung des politischen Projektes gegen die Ehe für alle diskutieren wir entlang zweier Aspekte: Die strukturelle Kontur dieses Projekts wird zunächst anhand einer detaillierten Analyse des Politikprozesses bis zur Verabschiedung der Ehe für alle beschrieben. Der Blick auf die diskursive Kontur erfasst die Argumentationen von GegnerInnen der Ehe für alle anhand einer diskursanalytischen Untersuchung ausgewählter christlich-rechter und neurechter Medien. In der Zusammenführung beider Aspekte entwickeln wir abschlieBend eine Bewertung des politischen Projektes und diskutieren die Bedeutung seiner Formierung aus hegemonietheoretischer Sicht. 


\section{Die Ehe für alLe als Untersuchungsgegenstand AN DER SCHNITTSTELLE VON GESCHLECHTER- UND RECHTSEXTREMISMUSFORSCHUNG}

Wir betrachten die Debatten um die Öffnung der Ehe vor dem Hintergrund von Debatten um einen ,autoritären Sog“ (Eitel 2018), der vielerorts auch als ,,völkischautoritärer Rechtsruck“" (Häusler 2018) diskutiert wird (kritisch dazu Birsl 2019: 6). Damit verorten wir die Debatten um die Ehe für alle als Forschungsgegenstand explizit an der Schnittstelle zwischen Geschlechter- und Rechtsextremismusforschung.

Die beiden vergleichsweise jungen Forschungsfelder haben sich in den vergangenen Jahren verstärkt der Relevanz antifeministischer, gegen die Vielfalt sexueller und geschlechtlicher Lebensweisen gerichteter Diskurse und Mobilisierungen zugewandt (exemplarisch Hark/Villa 2015; Kuhar/Paternotte 2017; Lang/Peters 2018). Ausgangspunkt vieler Überlegungen ist die Beobachtung, dass insbesondere die rechtliche Liberalisierung einst starrer geschlechtlicher und sexueller Identitäten in den vergangenen Jahren eine Vielzahl an Gegenmobilisierungen evoziert hat (für Deutschland: u.a. Billmann 2015; für Frankreich: Paternotte 2018; für Europa: Kováts/Põim 2015). Getragen wurden diese von einem Spektrum aus christlichrechten und sogenannten neurechten Gruppierungen. In der politischen Agenda der Akteursgruppen finden sich ideologische Versatzstücke, die auf ,das heterosexuelle Familienmodell, die strikte Ablehnung der Gleichstellung anderer Lebensweisen und damit der sexuellen Selbstbestimmung“ (Reinfeldt 2014) abheben und dabei eine gewisse inhaltliche Flexibilität und Anschlussfähigkeit an andere gesellschaftliche Debatten aufweisen.

Die Begriffe neurechts sowie Neue Rechte werden widersprüchlich verwendet. Als Eigenbezeichnung, die das Attribut neu nicht lediglich in Verhältnis zum zeitlichen Auftauchen dieser Denkströmung verwendet, findet der Begriff Verwendung in einem politischen Spektrum, das sich in Abgrenzung zum bürgerlichen Konservatismus auf der einen und der völkischen neonazistischen Rechten auf der anderen Seite verortet (Langebach/Raabe 2016: 572). Unter Berufung auf hegemonietheoretische Überlegungen nach Gramsci sieht die Neue Rechte ihr Hauptbetätigungsfeld im Bereich der Metapolitik: „Auf diesem Feld der Sinngebung sucht die Neue Rechte durch eine Revolutionierung der in einer Gesellschaft vorherrschenden Überzeugungen (,Kulturrevolution von rechts') hegemonial zu werden, um damit überhaupt erst die Voraussetzungen zu schaffen für die zukünftige Eroberung der politischen Macht.“ (Kellershohn 2017) Dabei kommt ihr gerade in ihrer nach außen behaupteten Abgrenzung gegenüber konservativen und extrem rechten Milieus eine entscheidende Scharnierfunktion zu, weil sie in dem Versuch, eine diskursive Vorherrschaft zu erreichen, thematische Brücken zwischen den verschiedenen, im 
weitesten Sinne als rechts zu bezeichnenden Spektren schlägt (Gessenharter 1990: 66f). Jüngere Arbeiten ergänzen dies im Hinblick auf Geschlechter- und Familienpolitiken rechter Akteure: So kommt insbesondere den Mobilisierungen gegen ,Gender'-Politiken eine Scharnierfunktion zwischen unterschiedlichen konservativ und extrem rechten Spektren zu (exemplarisch Kovats/Põim 2015).

Wir machen auch die christliche Rechte als Akteursgruppe aus, die an der Vorstellung einer Ungleichwertigkeit der Geschlechter festhält. In Deutschland lassen sich unter diesem Begriff sowohl evangelikale, protestantisch-fundamentalistische als auch ultrakatholische Gemeinschaften zusammenfassen, die auf die Beeinflussung öffentlicher Debatten im Sinne tradierter christlicher Ordnungsvorstellungen hinarbeiten (Teidelbaum 2018). Im Unterschied zur Neuen Rechten werden die darin zum Ausdruck kommenden antidemokratischen und antipluralistischen Ideen nicht biologistisch oder philosophisch, sondern durch den Bezug auf religiöse Glaubenssätze gerechtfertigt (Birsl 2018). Trotz dieses andersgelagerten Begründungszusammenhangs weisen Positionen der christlichen Rechten eine inhaltliche Nähe zu Standpunkten der Neuen Rechten auf.

Wir begreifen die geschlechterpolitische Agenda dieser Gruppen in ihrem Kern als antifeministisch und autoritär-regressiv: antifeministisch, da sie dem gleichstellungspolitischen Anliegen einer Entnormierung und Liberalisierung der Geschlechterverhältnisse entgegensteht. Beide Aspekte sind für uns die zentralen Merkmale eines weitgefassten, herrschaftskritischen Feminismusbegriffs, der sich in seinem Emanzipationsstreben grundsätzlich für die Aufhebung aller Ungleichheitsverhältnisse einsetzt (Lang/Fritzsche 2018: 340). Das antifeministische Projekt positioniert sich also nicht nur gegen konkrete feministische Forderungen, sondern grundsätzlich gegen jede Form geschlechtlicher und sexueller Selbstbestimmung, wodurch es sich etwa von einer konservativen Feminismuskritik unterscheidet (vgl. Lenz 2018).

Das Attribut autoritär beschreibt zunächst das grundlegende Politik- und Gesellschaftsverständnis des Projektes, welches die einzelnen Individuen dazu auffordert, sich vorbehaltslos verschiedenen sozialen Zwängen zu unterwerfen (vgl. auch: Lang 2019). Neue Arbeiten aus Sozialpsychologie (Decker/Brähler 2018) und Sozialwissenschaften (Heitmeyer 2018) charakterisieren dies als Teil einer Autoritarisierung von Politik und Gesellschaft. Im Rahmen unserer Fallstudie zeigt sich ganz konkret, dass die Politikangebote des von uns untersuchten Projektes für hierarchisch strukturierte Kontroll- und Disziplinierungsideen ${ }^{4}$ werben, die auf eine Retraditionalisierung der sich pluralisierenden Geschlechter- und Sexualitätsverhältnisse zielen. Die Bezeichnung als „regressiv“ verweist auf sozialtheoretischer Ebe-

4 Den Aspekt der „Kontrollparadigmen“ betont auch der Soziologe Wilhelm Heitmeyer (2018: 249ff) in seiner Konzeption des ,autoritären Nationalradikalismus“, mit der er den gegenwärtigen gesellschaftlichen Rechtsruck analytisch zu fassen versucht. 
ne darauf, dass die vorgebrachten Forderungen des Projektes „hinter das in der sozialen Moderne erreichte Niveau gesellschaftlicher Integration zurückfallen“ (Nachtwey 2016: 75), also institutionalisierte Errungenschaften einer progressivliberalen Gleichstellungspolitik zurücknehmen oder grundlegend umgestalten wollen. Denn anti-modern im eigentlichen Sinne sind die im Antifeminismus vertretenen Geschlechter- und Sexualitätsverhältnisse keineswegs. Sie werben für eine andere Moderne, in der traditionell heteronormative Geschlechterordnungen das Stützungsverhältnis einer autoritär-regressiven, illiberalen Gesellschaftsordnung bilden.

Diese Dynamik ist im internationalen Vergleich kein Einzelfall - Aktivist_innen wie Autor_innen aus verschiedenen Ländern berichten davon, dass sich ,als Reaktion auf einige LGBTIQ*-Erfolge eine immer stärker werdende Gegenbewegung etabliert" hat und sprechen in dem Zuge von einer ,globale[n] rechtsnationale[n] Bewegung[, die] die wachsende Akzeptanz von Homosexualität zunehmend als Anlass [nutzt], einen Kulturkampf zu führen“ (Schreiber 2018: 11).

Die Ehe für alle als Untersuchungsgegenstand an der Schnittstelle zwischen Geschlechter- und Rechtsextremismusforschung zu betrachten lenkt den Blick auf die rechte GegnerInnenschaft zur Eheöffnung. Aus dem Blick geraten dabei Positionen, die auf eine Kritik der Ehe als patriarchales Institut abzielen - und sich nicht im von uns untersuchten politischen Projekt wiederfinden. Sie spielen im Politikprozess durchaus eine Rolle, wie unsere Interviews zeigen. Wir kommen an gegebener Stelle darauf zurück.

\section{Kampf um Hegemonie: ZUM HISTORISCH-MATERIALISTISCHEN ANALYSEANSATZ}

Die von uns analysierten Auseinandersetzungen um die Einführung der Ehe für alle bewegen sich an der Schnittstelle zwischen verschiedenen gesellschaftlichen Herrschaftsverhältnissen und Konfliktfeldern. Unmittelbar berührt sind die Geschlechter- und Sexualitätsverhältnisse, es geht jedoch auch grundsätzlich um Vorstellungen zur Ordnung der Gesellschaft als solcher.

\subsection{Geschlechter- und Sexualitätsverhältnisse als gesellschaftliche Stützungsverhältnisse}

Die klassische Regulationstheorie betrachtet das Zusammenspiel gesellschaftlicher Verhältnisse in kapitalistischen Gesellschaften zunächst als eine Form des Akkumulationsregimes. Unter Akkumulationsregime versteht sie ein historisch und räumlich spezifisches Zusammenspiel aus Produktions- und Lebensweise, verbunden mit einer spezifischen Form staatlicher Herrschaft (Hirsch 1995: 49). Dieses 
Verhältnis ist auf eine konkrete Weise reguliert, welche die im Akkumulationsregime vorhandenen Widersprüche abschwächt und die Gesellschaft insgesamt temporär stabilisiert. Dieser Regulationsmodus ist mit einer spezifischen hegemonialen Struktur, also einer institutionalisierten Rechtfertigungsordnung verbunden (Lipietz 1985: 121). Aus geschlechterreflektierender Perspektive geht es darum, die Geschlechter- und Sexualitätsverhältnisse dabei systematisch mit in den Blick zu nehmen und den regulationstheoretischen Zugang analytisch zu erweitern (Kohlmorgen 2004).

Der Regulationsmodus lässt sich als ein Zusammenspiel verschiedener Faktoren denken, die zusammen das gegenwärtige Regulationsregime stützen. Wenn wir davon sprechen, dass der Konflikt um die Ehe für alle verschiedene gesellschaftliche Felder berührt, betrachten wir diese Felder als solche Stützungsverhältnisse (Dück 2014). Hierzu zählen etwa Geschlechter- und Sexualitätsverhältnisse, Produktionsund Reproduktionsverhältnisse sowie die Rechtsordnung. Am Beispiel der Ehe lässt sich dieser Zusammenhang gut aufzeigen. Die Ehe als Institution war bis zu ihrer Öffnung symbolischer Ausdruck der heteronormativen Geschlechterordnung mitsamt ihren Ausschlüssen. Gleichzeitig war sie auch die privilegierte Form des Zusammenlebens als Familie und somit Ausdruck einer sozialen Struktur, welche die Familie als Quelle des Gemeinwesens versteht (Notz 2015). Die im Zuge der Industrialisierung innerhalb der heterosexuellen Kleinfamilie etablierte geschlechtsspezifische, asymmetrische Arbeitsteilung mit ihren unterschiedlichen Bewertungslogiken von ,produktiver' und ,reproduktiver' Arbeit ist Grundlage für das Funktionieren aller bisher bekannten kapitalistischen Regulationsmodi, da sie die Abdrängung größtenteils von Frauen unbezahlt oder prekär geleisteter Reproduktionsarbeiten (Kindererziehung, Pflege etc.) ins ,Private ermöglicht. $^{5}$ Diese Funktion von Familie lässt sich in unterschiedlichen Wohlfahrtsregimes beobachten.

Auch wenn sich die Ehe somit als ein Knotenpunkt gesellschaftlicher Stützungsverhältnisse beschreiben lässt, nimmt dieser abhängig vom historischen und soziopolitischen Kontext eine unterschiedliche Form an. So lassen sich im Übergang zwischen den Regulationsregimen Fordismus und Postfordismus Änderungen im konkreten Zusammenspiel gesellschaftlicher Stützungsverhältnisse beobachten. Für unsere Analyse ist wichtig, dass Regulation immer eine diskursive Rechtfertigung braucht und das vor allem angestrebte Transformationen und Neukonfigurati-

5 Gleichzeitig kann Familie als Versorgungsgemeinschaft auch ein potenzieller Ort für Widerstand gegen patriarchale Strukturen und kapitalistische Arbeitsteilung sein. Es geht also nicht darum, Familie als solche zu problematisieren, sondern eine spezifische historische Version dieser, die vor allem konservative und rechte Diskursstränge als quasi ,naturgegeben" beschreiben. 
onen der Stützungsverhältnisse durch neue diskursive Muster begleitet werden müssen, wenn ein neuer hegemonialer Konsens erreicht werden soll.

\subsection{Stützungsverhältnisse als Orte von Kämpfen um Hegemonie}

Konkrete Kämpfe um (neue) Hegemonien entstehen, wenn sich strukturelle Veränderungen in den einzelnen Stützungsverhältnissen vollziehen oder diese von einzelnen Akteursgruppen diskursiv angestrebt werden. So stellt die Debatte um die Einführung der Ehe für alle zwar nicht die gesellschaftlichen Geschlechter- und Sexualitätsverhältnisse als solche in Frage, fügt sich jedoch in gegenwärtige Liberalisierungstendenzen ein, welche die Stützungsverhältnisse zu verändern scheinen. Die Geschlechtersoziologin Ilse Lenz deutet diese Veränderung als den Übergang von einer bisher hegemonialen differenzbasierten hin $\mathrm{zu}$ einer flexibilisierten Geschlechterordnung und macht dies u.a. daran fest, dass Geschlecht zunehmend „nicht mehr als biologisch bestimmtes Schicksal, sondern eher als Frage variierender persönlicher Lebensentwürfe aufgefasst“" werde (Lenz 2013: 129). Dieser Übergang, in dem die heteronormative Geschlechter- und Sexualitätsordnung etwas von ihrer vermeintlichen Selbstverständlichkeit verliert, berührt den gegenwärtigen Regulationsmodus als solchen, indem er die sich vollziehende Auflösung der fordistischen Kleinfamilie mit ihrer geschlechtsspezifischen Arbeitsteilung verstärkt und neue hegemoniale Deutungskämpfe ankündigt. Im Bereich des Wissens über die Geschlechter und die Rechtsordnung ist es (queer-)feministischen Bewegungen gelungen, die Fragen aufzuwerfen, welche sexuellen bzw. geschlechtlichen Identitätsentwürfe Teil der gesellschaftlichen ,Normalität' sein können und welche Formen staatlicher Anerkennung und Privilegierung diese Identitätsentwürfe erfahren sollen. Auch wenn diese Dynamik noch nicht die Geschlechter- und Sexualitätsverhältnisse als solche grundlegend erschüttert (Henninger/Backöfer/Fritzsche/NäserLather in diesem Band), trägt sie doch dazu bei, die Geschlechterordnung als im Fluss erscheinen zu lassen. Gegen dieses Anliegen emanzipatorischer Bewegungen formieren sich Positionen, welche mithilfe kampagnenartiger Interventionen die Wiedergewinnung der Deutungsmacht rund um die Themen Familie und Geschlecht im Sinne einer Retraditionalisierung anstreben. Dabei arbeiten sie mit konstruierten Bedrohungsszenarien und feldspezifischen Krisenszenarien wie der ,Krise der Männlichkeit‘ oder der ,Krise der deutschen Familie‘. Sie nutzen diese Diagnosen nicht zuletzt als Strategien zur Resouveränisierung des männlichen Subjekts und der patriarchalen Geschlechterordnung (Forster 2006). 


\subsection{Der Ansatz der Historisch-Materialistischen Politikanalyse}

Als Analyseinstrument für Kämpfe um Hegemonie in Debatten um die Ehe für alle dient uns die Historisch-Materialistische Politikanalyse (HMPA). Auf staatstheoretischen Erweiterungen Gramscis aufbauend (Brand 2013) und insbesondere in der kritischen Europaforschung weitergedacht (Becker et al. 2013), konzeptionalisiert die HMPA gesellschaftliche Herrschaftsverhältnisse in hegemonietheoretischer Perspektive als Ausdruck sozialer Kämpfe im ,integralen Staat“ (Becker et al. 2013: 68ff). Der Blick richtet sich damit sowohl auf die Zivilgesellschaft als auch auf staatliche Institutionen. In beiden Bereichen findet ein Ringen politischer Interessengruppen um die Deutungshoheit in zeitgenössischen Debatten statt. Dies umfasst den Versuch, neue hegemoniale Bündnisse und Kompromisse zu schmieden - und damit (regulationstheoretisch gedacht) um die Legitimation und Konfiguration der Stützungsverhältnisse und des Regulationsmodus zu ringen. Angestrebt wird ein Zustand der Hegemonie, in dem die „regierende Macht Zustimmung zu ihrer Herrschaft von denen erheischt, die sie unterjocht“ (Eagleton 2000: 133). Die gramscianische Definition von Hegemonie als Zusammenspiel von Konsens und Zwang verweist auf die ambivalenten Modi und Eigenlogiken, über die sich dieser Zustand im integralen Staat herstellen kann.

In Übereinstimmung mit anderen Forschungsarbeiten (Wolf 2016; Syrovatka 2016; Meißner 2017) nehmen wir an, dass der Analyserahmen der HMPA auf verschiedene politische Felder und Untersuchungsgegenstände angewandt werden kann, solange zentrale Annahmen beibehalten werden oder ihre Modifikation nachvollziehbar begründet wird. Besonders relevant für unsere Adaption der HMPA ist die Einsicht der feministischen Staatstheorie, dass der Staat als „,materielle Verdichtung gesellschaftlicher Kräfteverhältnisse" (Poulantzas 2002: 154) immer auch die Geschlechter- und Sexualitätsverhältnisse miteinschließen muss (Sauer 2001). Wie Buckel et al. (2014: 29) schreiben, weist die moderne Staatlichkeit eine „geschlechtsspezifische strategische Selektivität auf", die wiederum vergeschlechtlichte, aber auch ethnisierte Subjekte hervorbringt (Ludwig 2011: 193). Insofern ist es nur naheliegend, dass auch Geschlechterverhältnisse (und eng damit verbunden Sexualitätsverhältnisse) als Stützungsverhältnisse zeitgenössischer Herrschaftsformen zu einem Schauplatz von Kämpfen um Hegemonie werden.

Als anregend für unser Vorhaben betrachten wir die für die HMPA zentrale Annahme, dass einander nahestehende politische Akteure innerhalb der hegemonialen Auseinandersetzungen „begrenzte, konkrete politische Vorhaben“ verfolgen, um über die Durchsetzung dieser „politischen Projekte“ (Buckel et al. 2014: 48) langfristig hegemoniefähig zu werden. Regulationstheoretisch gesprochen würde dies heißen, dass Akteure sowohl die Deutungshoheit über einzelne Stützungsverhältnisse anstreben, als auch einen langfristigen gesamtgesellschaftlichen Ordnungsent- 
wurf (Regulationsmodus) zu etablieren versuchen. Dies ist für unseren Untersuchungsgegenstand besonders relevant, da die betrachteten GegnerInnen der Ehe für alle eher aus einer gesellschaftlichen Opposition heraus agieren und erst nach der Erringung von Hegemonie streben. Dabei legen wir den Fokus zunächst auf den Aspekt der diskursiven Formierung eines politischen Projektes, der dem der Durchsetzung zeitlich vorausgehen muss. Unter Formierung fassen wir die Phase, in der lose verbundene oder auch noch vereinzelte politische Akteure versuchen, ein spezifisches Verständnis konkreter sozialer Phänomene zu etablieren, um aufbauend auf diesem Narrativ oder dieser Problemdefinition weiterführende Handlungsstrategien oder Lösungsansätze vertreten zu können. Der Prozess der Außenkommunikation, der bewusst darauf zielt, die politischen Positionen des entstehenden Projektes in gesellschaftliche Auseinandersetzungen hineinzutragen, Bündnispartner_innen zu gewinnen und gegnerische Standpunkte zu destabilisieren, ist dabei bereits in der von uns untersuchten Anfangsphase eines politischen Projektes zu beobachten.

\section{Methodisches Vorgehen}

Wir betrachten die HMPA als Methodologie, der wir den zentralen Begriff des politischen Projekts für unsere Untersuchung entnehmen. Bei unserem analytischen Vorgehen lassen wir uns von dem diskurshistorischen Ansatz von Reisigl und Wodak (2001) anleiten, der es ermöglicht, unsere Forschungsfrage nach der Formierung eines politischen Projektes mithilfe einer Methodentriangulation sowohl auf struktureller als auch auf diskursiver Ebene zu verfolgen. Die Frage nach den strukturellen Konturen des Projektes berücksichtigt, dass dieses sich auf konkrete Akteursnetzwerke zurückverfolgen lassen muss, welche mit spezifischen Ressourcen an der Durchsetzung ihrer Ziele arbeiten. Die Frage nach diskursiven Konturen nimmt in den Blick, dass sich das Ringen um Hegemonie immer in konkreten Deutungs- und Legitimationskämpfen vollzieht und dass diese Diskurse darüber an der Reproduktion oder Veränderung der sozialen Wirklichkeit beteiligt sind (Reisigl/ Wodak 2001: 40).

\subsection{Erhebung der strukturellen Kontur des politischen Projekts}

Wir sind in einem insgesamt vierstufigen Verfahren vorgegangen: Zunächst bestimmten (1) wir anhand von Sekundärliteratur und bereits vorliegenden Analysen den Kontext des Untersuchungsgegenstandes, rekonstruierten den politischen Prozess bis zur Verabschiedung der Ehe für alle und identifizierten die relevanten Akteure im Feld. Mithilfe einer Dokumentenanalyse betrachteten wir (2) den Diskursverlauf, um den Verlauf, zentrale Akteure und diskursive Ereignisse zu verifizieren 
und relevante Dokumente als empirisches Material zu identifizieren. Hierfür durchsuchten wir die Protokolle von Bundestagsdebatten und Drucksachen des Bundestags zu den daran anschließenden Entscheidungen sowie einschlägige Urteiles des Bundesverfassungsgerichtes.

Auf der Grundlage der Erkenntnisse aus der Dokumentenanalyse befragten wir (3) außerparlamentarische Beteiligte am politischen Prozess zur Ehe für alle. Diese Interviews mit Expert_innen aus der lesbisch-schwulen Community trugen bewusst einen explorativ-felderschließenden Charakter, um das breite Kontextwissen der Interviewpartner_innen über die 25-jährige Kontroverse um die Ehe für alle einzufangen. Nach Meuser und Nagel ,resultiert die Perspektive auf Kontextwissen von Expert_innen aus der Betrachtung eines Sachverhaltes, an dessen Zustandekommen nicht nur, sondern auch die Expertlnnen maßgeblich beteiligt sind“ (Meuser/Nagel 2005: 76). Es ging hier also um Akteure der Zivilgesellschaft, die im außerparlamentarischen Raum um die Berechtigung gleichstellungspolitischer Anliegen und damit um die Frage des ,richtigen“ Deutungswissens rangen. Im Zentrum dieser Expert_inneninterviews mit Vertreter_innen von LGBTIQ*-Organisationen stand das Deutungswissen um ein geschlechterpolitisches Liberalisierungsprojekt, welches mit den Beschlüssen von Bundestag und Bundesrat Realität wurde. Insgesamt wurden drei Expert_inneninterviews zum Kontextwissen durchgeführt.

Zusätzlich erhoben wir (4) das Betriebswissen (Meuser/Nagel 2005) der am parlamentarischen Prozess beteiligten Bundestagsabgeordneten. In insgesamt neun Interviews mit Abgeordneten aller im 18. Deutschen Bundestag (2013-2017) vertretenen Parteien erhoben wir Perspektiven zum Verlauf des parlamentarischen Prozesses. Wir fragten u.a. nach dem Einfluss eines von uns außerparlamentarisch verorteten politischen Projektes gegen die Eheöffnung auf Debatten im Parlament. ${ }^{6}$ Trotz Ansprache von Parlamentarier_innen aller Fraktionen, die in der Debatte um die Ehe für alle unterschiedlich positioniert und eingebunden waren, erhielten wir nur Rückmeldungen von Abgeordneten, die am 30. Juni 2017 im Bundestag für die Ehe für alle gestimmt haben.

6 CDU (2), CSU (1), SPD (2), LINKE (2) und Grüne (1) sowie ein Vertreter einer lesbischschwulen Parteiunterorganisation. Wir wandten uns zunächst an die jeweiligen themenspezifischen Sprecher_innen der einzelnen Fraktionen (queer- und familienpolitische Sprecher_innen) und wurden von diesen teilweise weiterverwiesen. Ein_e der IP sitzt erst seit 2017 im Deutschen Bundestag, ist dort jedoch in verantwortlicher Position für den von uns untersuchten Themenbereich zuständig. 


\subsection{Erhebung der diskursiven Kontur des politischen Projekts}

Nachdem die Schritte zur Erschließung der strukturellen Dimension bereits erste Hinweise auf das diskursive Agieren verschiedener GegnerInnen der Ehe für alle geliefert hatten, wurde dieses näher untersucht. Unsere Annahme war, dass sich ein politisches Projekt neben strukturellen Verknüpfungen auch durch interdiskursive Verbindungen auszeichnen müsse, also etwa durch geteilte Argumentationsmuster, gegenseitige Bezugnahmen einzelner Sprecher_innen aufeinander oder durch gemeinsame diskursive Interventionen. Diese Elemente sind notwendige Bestandteile für die Entwicklung eines geteilten Deutungsrahmens und dessen Kommunikation nach außen. Hier galt es zu bestimmen, welche dieser Elemente sich in neurechten und christlich-rechten Internetportalen wiederfinden.

Bei der analytischen Annäherung an die Texte unterschieden wir in Anlehnung an den diskurshistorischen Ansatz drei Analysedimensionen: Inhalte, Argumentationsstrategien und -figuren sowie sprachliche Realisierungsmittel (Wodak/Köhler 2010: 37). In ersten Analyserunden entwickelten wir mithilfe der qualitativen Analysesoftware MAXQDA ein Kategoriensystem für das Material. ${ }^{7}$

Der Auswahl der Online-Medien für unsere diskurs- und argumentationsanalytische Auswertung lagen drei Kriterien zugrunde. Wir wollten (1) die mögliche thematische Varianz der Positionen gegen die Ehe für alle im neurechten und christlich-rechten Spektrum abbilden, auf die die Sekundärliteratur sowie die vorhergehenden Interviews und die Dokumentenanalyse hinwiesen. Die jeweiligen Medienorgane sollten (2) über eine gewisse diskursive Reichweite verfügen, da sich die potenzielle Zugehörigkeit zu einem politischen Projekt über das Ziel der Hegemoniefähigkeit definiert. Dies setzt voraus, dass zumindest vom Anspruch her der Versuch unternommen wird, an gesellschaftlichen Debatten jenseits des eigenen politischen Milieus teilzunehmen. Schließlich sollten (3) die jeweiligen Organe aus forschungspraktischen Erwägungen über ein klassisches Online-Archiv verfügen, oder sich mithilfe einer Suchfunktion nach thematischen Beiträgen durchforsten lassen. Auf Grundlage dieser Kriterien entschieden wir uns für vier Medien: Die Wochenzeitung Junge Freiheit sowie die drei Internetblogs Freie Welt, kath.net und Charismatismus. Damit sind das neurechte und das christlich-rechte Spektrum mit jeweils zwei Online-Medien bzw. Internetblogs vertreten.

Die Junge Freiheit ist eine neurechte Wochenzeitung, die seit ihrer Gründung 1986 im Grenzbereich zwischen Konservatismus und Rechtsextremismus agiert

7 Wir griffen hier auf Vorarbeiten wie das Codebuch der Master-Arbeit von Anne Gehrmann (2018) zurück und ergänzten gleichzeitig induktiv neue Kategorien. Das erweiterte Kategoriensystem kann zu Forschungszwecken unter der Email-Adresse reverse@staff. uni-marburg.de angefragt werden. 
(Gessenharter 1989: 426). Auch wenn sich die Zeitung gegenwärtig vom völkischen Nationalismus in seiner offenen Form distanziert, gilt sie weiterhin als „Sprachrohr einer radikalnationalistischen Opposition, der es um eine fundamentale Veränderung der gesellschaftlichen, politischen und kulturellen Verhältnisse in Deutschland geht“" (Botsch 2017). Die Junge Freiheit ist aktuell die sechstgrößte deutsche Wochenzeitung und konnte ihre Reichweite gegen den Trend am Printmedienmarkt in den letzten Jahren stabilisieren (Schröder 2019). Die Druckauflage betrug im zweiten Quartal 2019 rund 31.500 Exemplare (IVW 2019), der OnlineAuftritt der Zeitung wird täglich etwa 190.000-mal angeklickt. ${ }^{8}$

Die Freie Welt ist eine 2009 als Teil des Kampagnennetzwerks Zivile Koalition von Beatrix und Sven von Storch gestartete Blogplattform. Sie soll heute als „Mosaik aus eigenen Medien, Plattformen und Vereinen [...] die konservativchristlichen Interessen sowohl in der gesamten Gesellschaft als auch in der AfD stärken“ (Fuchs/Middelhoff 2019: 119). Obwohl sich das Online-Medium als klassische Nachrichtenseite inszeniert, finden sich dort stark meinungsgefärbte Beiträge, die größtenteils den Positionen der AfD nahestehen (Linde 2014). Das Blog wird täglich etwa 25.000-mal aufgerufen.

Kath.net ist nach der Selbstbeschreibung ein „katholischer Nachrichtendienst“, der 1999 als privates Online-Magazin mit täglichen nachrichtenähnlichen Beiträgen gegründet wurde. Das Portal vertritt Standpunkte der christlichen Rechten und funktioniert laut Angelika Strube als „Scharnierorgan“ (Strube 2012), das neurechte Inhalte ,sehr bürgerlich aufbereitet“ (Deutschlandfunk 2014). Nach Strube (2017: 58) fungiert kath.net kampagnenartig als „Werbetrommel für die AfD“. Das Blog wird täglich etwa 25.000-mal angeklickt.

Das „christliche Forum“ Charismatismus wird seit 2011 von der katholischen Journalistin Felizitas Küble betrieben, die sich als Buchhändlerin und Publizistin hauptberuflich der Verbreitung evangelikaler und ultrakatholischer Positionen widmet. Küble schreibt des Weiteren für die Freie Welt, die Blogs Konservo und Kreidfeuer sowie The European und die Junge Freiheit. Die Publizistin wurde 2019 in einer Marktforschungsuntersuchung zu den erfolgreichsten deutschen Bloggerinnen auf Platz 10 gerankt, ohne dass ihr politischer Hintergrund dabei zur Sprache kam (marktforschung 2019). Die täglichen Aufrufe des Blogs werden nicht gezählt, es weist aber aktuell insgesamt über fünf Millionen Seitenaufrufe auf.

Bei der Eingrenzung des Untersuchungszeitraums halfen uns die Ergebnisse der vorhergehenden Dokumentenanalyse und der explorativ-felderschließenden Interviews. Der Lesben- und Schwulenverband in Deutschland (LSVD) entschied sich

8 Alle im Folgenden referierten Daten zur Online-Reichweite der untersuchten Medien wurden mithilfe des Online-Dienstes Wolframalpha (https://www.wolframalpha.com/) generiert und geben die Datenlage im Oktober 2019 wieder. 
zehn Jahre nach Einführung der eingetragenen Lebenspartnerschaft im Sommer 2011 dazu, offen die Öffnung der Ehe zu fordern. Damit nahm der politische Prozess erneut an Fahrt auf. Unser Untersuchungszeitraum beginnt somit im Jahr 2011 und endet mit dem Oktober 2017, dem Zeitpunkt des Inkrafttretens des Gesetzes zur Ehe für alle. Innerhalb dieses zeitlichen Rahmens durchsuchten wir die OnlineArchive der ausgewählten Medien nach thematischen Beiträgen, die in der Schlagwort- und/oder Volltextsuche den Begriff Homo-Ehe oder Ehe für alle beinhalteten. ${ }^{9}$ Dieser erweiterte Korpus bildete zunächst die Grundgesamtheit unserer Untersuchung.

Tabelle 1: Grundgesamtheit des Textkorpus

\begin{tabular}{|l|c|c|c|c|c|c|c|c|}
\hline Medium/Jahr & $\mathbf{2 0 1 1}$ & $\mathbf{2 0 1 2}$ & $\mathbf{2 0 1 3}$ & $\mathbf{2 0 1 4}$ & $\mathbf{2 0 1 5}$ & $\mathbf{2 0 1 6}$ & $\mathbf{2 0 1 7}$ & SUM \\
\hline Charismatismus & 1 & 3 & 60 & 9 & 37 & 11 & 47 & 195 \\
\hline Freie Welt & 1 & 38 & 46 & 15 & 40 & 15 & 16 & 141 \\
\hline Junge Freiheit & 18 & 26 & 65 & 24 & 39 & 10 & 51 & 233 \\
\hline Kath.net & 19 & 44 & 93 & 43 & 81 & 25 & 103 & 408 \\
\hline Summe & 39 & 108 & 264 & 91 & 197 & 61 & 217 & 977 \\
\hline
\end{tabular}

Dieser Korpus enthielt Textsorten aller Art - von kurzen Meldungen, in denen etwa der Begriff Homo-Ehe nur am Rand auftauchte, bis hin zu umfangreichen Kommentaren, die unsere Forschungsfrage berührten. Bei der thematischen Eingrenzung sortierten wir zunächst alle Texte aus, die sich nicht vorrangig mit der Debatte um die Einführung der Ehe für alle in der Bundesrepublik befassten. Wir beließen außerdem nur Beiträge im Korpus, die über eine bloße Nennung der Begriffe hinausgehen und die Homo-Ehe bzw. Ehe für alle in die eigene Argumentation einbeziehen. Nach dieser Bereinigung der Grundgesamtheit erhielten wir einen reduzierten Korpus (Tabelle 2).

9 Im französischen Wahlkampf 2012 hatte der Sozialist François Hollande den Begriff marriage pour tous in seinen Wahlversprechen verwendet, die Ehe mit all ihren Rechten und Pflichten für gleichgeschlechtliche Paare zu öffnen. Dies betraf insbesondere Fragen der Adoption von Kindern, in denen Ehe und eingetragene Lebenspartnerschaft bis dato nicht gleichgestellt waren. Seit 2013 fand auch in Deutschland mehr und mehr der Begriff der Ehe für alle Verwendung. Es ist nicht eindeutig festzumachen, wann und durch wen der bis dato verwendete Begriff Homo-Ehe abgelöst wurde. 
Tabelle 2: Bereinigter Textkorpus

\begin{tabular}{|l|c|c|c|c|c|c|c|c|}
\hline Medium/Jahr & $\mathbf{2 0 1 1}$ & $\mathbf{2 0 1 2}$ & $\mathbf{2 0 1 3}$ & $\mathbf{2 0 1 4}$ & $\mathbf{2 0 1 5}$ & $\mathbf{2 0 1 6}$ & $\mathbf{2 0 1 7}$ & SUM \\
\hline Charismatismus & - & 1 & 21 & 2 & 5 & - & 9 & 51 \\
\hline Freie Welt & - & 3 & 15 & 2 & 1 & 1 & 2 & 37 \\
\hline Junge Freiheit & 4 & 4 & 7 & - & 1 & 1 & 2 & 30 \\
\hline Kath.net & 1 & 2 & 9 & 2 & 4 & 3 & 1 & 31 \\
\hline Summe & 5 & 23 & 52 & 6 & 34 & 5 & 23 & 148 \\
\hline
\end{tabular}

\section{Die EHE FÜR ALLE UND IHRE GEGNERINNEN: EIN GesellschaftSPOLITISChER PROZESS}

Als die Ehe für alle im Sommer 2017 von Bundestag und Bundesrat verabschiedet wurde, zeigten sich mediale Kommentator_innen überrascht darüber, dass das Gesetz den Bundestag letztlich so schnell passiert hat. ${ }^{10}$ Dagegen verweisen sämtliche unsere Gesprächspartner_innen auf die jahrelangen Vorarbeiten und insbesondere die Kämpfe der schwul-lesbischen Community und der politischen Mitstreiter_innen. Unsere Interviewpartner_innen bekunden fast übereinstimmend: Die Gesellschaft war einfach soweit. Sie beziehen sich auf Umfragen aus den Jahren 2015/2016 und 2017, ${ }^{11}$ in denen sich knapp 80 Prozent der Befragten für die Öffnung der Ehe aussprachen. Allen Konflikten zum Trotz sprechen die Interviewten parteiübergreifend davon, die Entscheidung zur Ehe für alle im Sommer 2017 habe die Debatte langfristig befriedet:

,[D]as ist selbst bei denjenigen, die jetzt nicht mit wehenden Fahnen für die Öffnung der Ehe gestimmt haben, die sagen auch das Thema ist jetzt durch, es ist auch gesellschaftlich befriedet, glaube ich auch, natürlich gibt's immer noch Leute, die das für eine falsche Entscheidung

10 So z.B. Heribert Prantl in der Hörfunk-Sendung des SWR2 (2017): „Die Abstimmung über die ,Ehe für alle““, in: Forum vom 30.06.2017. [https://www.ardmediathek.de/ radio/Forum/Die-Abstimmung-über-die-Ehe-füralle/SWR2/Audio?bcastId=8758428\& documentId=54047528; abgerufen am 23.03.2020].

11 Vgl. Umfragen des Meinungsforschungsinstitut YouGov aus den Jahren 2015/2016 (Schmidt 2015, 2016) sowie die Studie der Antidiskriminierungsstelle des Bundes Einstellungen gegenüber Lesben, Schwulen und Bisexuellen in Deutschland (2017). 
halten, aber man hat gemerkt, das christliche Abendland ist nicht untergegangen und auch nicht massenhaft heterosexuelle Ehe deshalb geschieden worden deswegen“ (Interview F).

\subsection{Der Politikprozess bis zur Ehe für alle}

Unsere Dokumentenanalyse zeigt: Mit dem Inkrafttreten des Gesetzes zur eingetragenen Lebenspartnerschaft wurde im Jahre 2001 von der rot-grünen Bundesregierung ein rechtlich verbindliches, ehe-äquivalentes Institut für gleichgeschlechtliche Paare geschaffen - wie vom Schwulenverband Deutschland (SVD), ab 1998 Lesben- und Schwulenverband Deutschland (LSVD), seit Langem gefordert. Von Politiker_innen mit Community-Anbindung, wie Volker Beck von Bündnis 90/Die Grünen, wurde diese Forderung seit Mitte der 1990er Jahre auch im Parlament vorgebracht. Teile der lesbisch-feministischen Community standen dem Anliegen skeptisch gegenüber:

„[D]er Lesbenring hat 'ne Denkwerkstatt, da wurde mehr darüber philosophiert, dass es nicht ok ist. Die Lebenspartnerschaft hilft nicht, Ehe für Alle hilft auch nicht, weil wir wollen nicht mehr in dieses Muster, wir wollen andere Beziehungen leben.“ (Interview III)

Die heterosexuelle Ehe wird von Akteuren wie dem Lesbenring e.V. in ihrer Stützungsfunktion heteronormativer Geschlechterungleichheit gelesen und kritisiert. Nicht die Öffnung der Ehe wird gefordert, sondern ihre Abschaffung als staatlich privilegierte Lebensform. Ehekritische Positionen insbesondere von Organisationen lesbischer Frauen (Davidson-Schmich 2017) begleiteten uns im Forschungsprozess. Auch Parlamentarier_innen der Parteien DIE LINKE und Bündnis 90/Die Grünen verweisen auf die Bedeutung ehekritischer Positionen in den parteiinternen Debatten, die jedoch bei keiner der Befragten dazu führten, die Öffnung der Ehe abzulehnen. Die Bedeutung, die der Ehe beigemessen wird, variiert jedoch stark.

„[I]ch bin jetzt sowieso nicht unbedingt die Eheverfechterin sondern für mich würd's auch gut ohne gehen. Ich fand aber natürlich auch immer so lange es dieses Institut mit seinen Privilegien und Vorrechten gibt muss es für alle gelten und nicht nur für heterosexuelle Paare. Dafür habe ich mich auch immer an entsprechenden Stellen eingesetzt“ (Interview C).

In der Anpassung homo- an heterosexuelle Familienmodelle gehe der emanzipatorische, queere Charakter ersterer verloren, so andere Kritiker_innen. Dies klingt auch mit im Vorwurf, die Eheöffnung sei im Kern kein progressiver Schritt gewesen. Dieser Vorwurf irritiert und verärgert einige derer, die sich seit Jahren für die Eheöffnung eingesetzt haben: 
„[W]ar es einfach so ein bisschen ärgerlich tatsächlich, dass dann Leute irgendwie gesagt haben, das ist ja gar kein progressives Anliegen, wenn 80\% der Menschen dafür sind, zeigt doch eigentlich wie sehr die Ehe in das heteronormative Modell schlechthin eingebaut werden kann, [...] ich find's tatsächlich dumm, also man kann sich darüber unterhalten, inwiefern Ehe als Anliegen wichtig ist für die LSBTI-Community und ob's nicht noch wichtigere Themen gegeben hätte, aber zu sagen, weil die Mehrheit der Bevölkerung dafür ist, zeigt's einfach nur wie zahnlos und bedeutungslos diese Anliegen eigentlich ist, erschließt sich mir in keinster Weise“ (Interview I).

Nicht nur das Lebenspartnerschaftsgesetz, sondern auch die Ehe für alle wurde und wird von ihren GegnerInnen stets als, rot-grünes‘ Projekt markiert. Die konservativ regierten Bundesländer Bayern, Thüringen und Sachsen scheiterten 2002 mit einer Normenkontrollklage gegen das Lebenspartnerschaftsgesetz vor dem Bundesverfassungsgericht. In den Folgejahren verlor der Prozess zur Gleichstellung hetero- und homosexueller Lebensgemeinschaften an Fahrt. Parlamentarisch wurden vorerst keine weiteren Initiativen gestartet. Dagegen ergingen im selben Zeitraum von höchster juristischer Instanz mehrere Urteile, welche die fehlende Gleichbehandlung eingetragener Lebenspartnerschaften anmahnten: u.a. in Bezug auf steuerliche Fragen und die Hinterbliebenenrente. Das Parlament wurde häufig nur dort tätig, wo die Gerichte einen expliziten Auftrag zur Beseitigung bestehender Ungleichbehandlungen gaben. Es handelt sich hierbei um ein demokratiepolitisches Dilemma, was sicher mit dazu beigetragen hat, dass die später verabschiedete Ehe für alle mitunter eher als „Projekt von oben“ denn als die Frucht jahrelanger Kämpfe gesehen wurde (Lang 2019).

Anlässlich von zehn Jahren Lebenspartnerschaftsgesetz gab es dann im Sommer 2011 eine Festveranstaltung des LSVD im Berliner Roten Rathaus, auf der gefordert wurde, statt schrittweiser Anpassungen fortan für die längst überfällige vollständige Öffnung der Ehe einzutreten. Dem folgten Aktivitäten des LSVD in den Jahren 2012 und 2013. Im Vorfeld der Bundestagswahlen 2013 versandte der LSVD Wahlprüfsteine an alle im Bundestag vertretenen Parteien sowie die Piratenpartei und forderte diese zu Positionierungen bezüglich der vollständigen Eheöffnung auf: Hierauf haben sich abgesehen von der Union alle Parteien für die Öffnung der Ehe ausgesprochen (vgl. Davidson-Schmich 2017). Die SPD machte die Eheöffnung 2013 zur Bedingung für eine erneute Koalition mit der Union - eine Position, die sie später belasten sollte: Interviewpartner_innen aus der Koalition wie auch Opposition sprechen von einem „Trauma“ sowie von „Glaubwürdigkeitsproblemen“ der SPD, die trotz anderslautender Bekundungen im Wahlkampf der Verschiebung der vorliegenden Anträge von Ausschusssitzung zu Ausschusssitzung zustimmte (exemplarisch Interview E). Entsprechende Gesetzesvorlagen von Bünd- 
nis 90/Die Grünen und der Partei DIE LINKE lagen seit 2009 vor, ${ }^{12}$ seit 2015 auch der Gesetzesentwurf des Bundesrats. ${ }^{13}$ Jedoch wurde die Beratung über den Gesetzesentwurf in den zuständigen Ausschüssen über zwei Jahre hinweg von Sitzung zu Sitzung vertagt.

\subsection{Mobilisierungen gegen die Ehe für alle}

Internationale Mobilisierungen für geschlechter- und insbesondere sexualitätsbezogene Liberalisierungen waren Ansporn für Befürworter_innen der Ehe für alle und seit 2013 vermehrt Anlass für Gegenmobilisierungen. Als erstes Land der Welt öffneten die Niederlande im Jahr 2000 die Ehe für gleichgeschlechtliche Paare. Fünfzehn Jahre später stimmte in Irland in einer Volksabstimmung eine Mehrheit für die Öffnung der Ehe, obwohl konservative und christlich-rechte Kreise viel Geld in Gegenkampagnen investiert hatten (Datta 2018). Konservative Politiker_innen wie Jens Spahn wurden in der Folge mit Aussagen zitiert, was Irland könne, könne man in Deutschland auch. Dies evozierte Reaktionen christlichrechter und neurechter Akteure, die dies als Anlass nahmen, ihre Ablehnung der Eheöffnung erneut zu begründen:

„Man huldigt derzeit den Iren für ihr Votum. Warum? Was ist an diesem Votum so überaus bedeutsam? Dient es etwa der Stabilität und dem Erhalt des Gemeinwesens? EHE beinhaltet nicht nur die , auf Dauer angelegte, rechtlich verfestigte Partnerschaft‘ zwischen zwei Menschen, sondern auch die Verantwortung für den Fortbestand der demografischen, der sozialen und der kulturellen Gesellschaft. [...] Ich bin sehr dafür, dass auch der Vatikan, etwas Neues lernen' kann und soll. Da gibt es Baustellen genug. Aber ich bin auch froh, dass er wegen eines irischen Referendums nicht bewährte Werte über Bord kippt.“ (Fischer 2015a, in Freie Welt vom 10.06.2015)

Das Jahr 2015 und insbesondere die Zeit rund um das irische Volksbegehren stellen einen Peak der Berichterstattung rund um sexuelle und geschlechtliche Vielfalt dar. Insbesondere für die neurechten Medien im Korpus - die Junge Freiheit und das Blog Freie Welt - dienten die Ereignisse des Jahres 2015 zur skandalisierenden Berichterstattung (Tabelle 2). Ein ähnlicher Peak zeigt sich bereits für das Jahr 2013:

12 Vgl. exempl. BT-Drs. 16/13596 der Fraktion Bündnis 90/Die Grünen vom 30.06.2009: „Entwurf eines Gesetzes zur Einführung des Rechts auf Eheschließung für Personen gleichen Geschlechts“; BT-Drs. 17/2023 der Fraktion DIE LINKE vom 09.06.2010, Antrag „Öffnung der Ehe“.

13 „Entwurf eines Gesetzes zur Einführung des Rechts auf Eheschließung für Personen gleichen Geschlechts“, BR-Drs. 273/15 sowie BT-Drs. 18/6665. 
Auch hier waren es internationale Ereignisse, auf die referiert wurde. Mit Neid blickten konservative, christlich und extrem rechte Kreise in Deutschland auf die Entwicklungen in Frankreich: Dort protestierten 2013 landesweit mehrere Hunderttausend Menschen unter dem griffigen Namen Manif pour tous (deutsch: Demo für alle) gegen die Pläne der französischen Regierung, das Adoptionsrecht für gleichgeschlechtliche Paare weiter zu öffnen. Vorgeblich ging es um die geplante Gesetzesnovelle - jedoch nutzten konservative und christliche Kreise die Proteste als Kritik an der Sozialistischen Regierung unter François Hollande und für ein Ringen um verloren geglaubte Hegemonie in Auseinandersetzungen um Ehe, Familie und die Ordnung der Geschlechter (Stambolis-Ruhstorfer/Tricou 2017). „Es ist zu wünschen, daß der Funke aus Frankreich überspringt und wir uns vom phantasievollen Protest und Widerstandsgeist unserer gallischen Nachbarn inspirieren lassen“, schrieb Dieter Stein, Chefredakteur der Jungen Freiheit, im Januar 2013 (Stein 2013, in Junge Freiheit vom 18.01.2013). Und die katholische Rechte Gabriele Kuby wünschte sich im Portal kath.net, ,was in Paris geschieht, sollte den Widerstand in ganz Europa ermutigen“" (Kuby 2013, in kath.net vom 18.01.2013).

Insgesamt erschienen in den von uns untersuchten Medien 2013 und 2015 jeweils mehr gegnerische Beiträge zur Ehe für alle als 2017, dem Jahr ihrer Verabschiedung. Die Debattenbeiträge zeugen von dem Versuch, nationale und internationale Dynamiken zu nutzen, in Debatten rund um die Gleichstellung hetero- und homosexueller Partnerschaften sowie die Öffnung der Ehe in Deutschland zu intervenieren. Der Zeitpunkt war jeweils günstig: So war 2013 zugleich das Jahr der Bundestagswahl, in deren Vorfeld der Diskussion um die Eheöffnung größere Aufmerksamkeit zu Teil wurde. 2015 nutzten auch Befürworter_innen die irische Volksabstimmung als Anlass, um für die Eheöffnung zu werben - und es gab somit eine Debatte zum Thema in der deutschen Öffentlichkeit.

\subsection{Ein heterogenes Akteursfeld im Kampf um Hegemonie}

In der Ablehnung der Eheöffnung und der damit verbundenen Verteidigung traditioneller Geschlechter- und Sexualitätsverhältnisse als gesellschaftliche Stützungsverhältnisse traf ein heterogenes Feld an Akteuren zusammen. Als Träger dieses politischen Projekts fungierten die christliche und Neue Rechte. Der Historiker Volker Weiß betont, wie sehr autoritäres (Geschlechter-)Denken ein heterogenes Akteursspektrum eint:

„Wie kaum ein anderes Beispiel stehen für die Überschneidung in der autoritären Weltanschauung, sei es in der politischen Rechten, sei es im religiösen Konservatismus, die rigiden Vorstellungen von Geschlechteridentität. Sie alle führen als Identitäre in ihrer Angst vor dem Nicht-Identischen einen wahren Feldzug gegen jede Form der Verunsicherung eines festen 
Geschlechterschicksals. Darin wurzelt die Todfeindschaft gegen alles, was diese Kategorien infrage stellt.“(Weiß 2017: 228)

Geschlechterverhältnisse, so führt es die Politikwissenschaftlerin Ursula Birsl (2017: 111) in Bezug auf die religiöse wie auch säkulare Rechte aus, sind als ,,konstitutiv für diese Weltanschauungen sowie deren Trägerinnen und Träger“" zu betrachten. Insbesondere in Fragen reproduktiver Rechte artikuliert ein lauterwerdender Teil christlich-rechter Akteure zunehmend anti-emanzipatorische Positionen und beteiligt sich selbstbewusst an Debatten um Geschlechter- und Sexualitätsverhältnisse. Birsl (2017: 113f) spricht von den dahinterstehenden politischen Theologien, als einem „restaurativem Denken“, welches eine moderne (religiöse) Ideologie hervorbringt (Birs1 2017: 115). Selbiges finden wir bei den von uns untersuchten Akteuren.

Die jeweiligen Akteursgruppen sind in Teilen eingebunden in den organisierten Antifeminismus. Hierüber erhalten sie Applaus von anderen Akteuren, wie maskulistischen MännerrechtlerInnen oder sog. ,WissenschaftskritikerInnen', die anders als die zuvor benannten jedoch nicht als TrägerInnen des politischen Projektes gegen die Ehe für alle ausgemacht werden können (vgl. auch Näser-Lather in diesem Band). Teile dieses Akteursspektrums finden sich als UnterstützerInnen von Kampagnen wie der Demo für alle. Von Februar 2014 an fand sich diese nach dem französischen Vorbild benannte Initiative in Stuttgart zusammen und protestierte zunächst gegen eine Bildungsplanreform der rot-grünen Landesregierung in BadenWürttemberg, welche auf die Anerkennung vielfältiger geschlechtlicher und sexueller Lebensweisen abzielte (Billman 2015). Die InitiatorInnen erweiterten schnell ihre thematische Agenda. Sie richteten ihre Mobilisierungen auch gegen die Eheöffnung und setzten sich für ein traditionell heteronormatives Verständnis von Familie ein. Die Proteste fanden bundesweit Resonanz. Die Akteure sind in Teilen bis heute aktiv mit ähnlich gelagerten Protesten, die sich unter dem Vorwurf einer angeblichen ,Frühsexualisierung ' oder der Warnung vor Menschenhandel durch Leihmutterschaft gegen die Ehe für alle richten (Datta 2018).

\section{6. „EIn PAPA, EINe Mama, Ganz EINFACH!" - DISKURSIVE KONTUREN EINES POLITISCHEN PROJEKTES}

Übergeordnetes Ziel des von uns untersuchten politischen Projektes ist es, eigenes Deutungswissen langfristig in gesellschaftlichen Debatten zu verankern - und Einfluss in Kämpfen um gesellschaftliche Hegemonie zu entfalten. Debatten um die Eheöffnung dienten und dienen hierfür als Anlass. Im Folgenden geht es darum, die diskursiven Konturen der (autoritär-regressiven) Gegnerschaft zur Ehe für alle auf- 
zuzeigen. Die diskurs- und inhaltsanalytische Untersuchung der bereits vorgestellten Medien bietet hierfür die Grundlage. In Beiträgen, die sich in Gänze kritisch gegen die Eheöffnung positionieren, erkennen wir medien- und akteursübergreifende Diskursstränge: thematische Verdichtungen rund um Vorstellungen von Ehe und damit verknüpft von Familie als alleinig heterosexueller Gemeinschaft sowie Fragen der Reproduktion und dem Verhältnis des analysierten politischen Projektes zum Staat. Hinzu kommt der hoch emotional und normativ aufgeladene Bezug auf

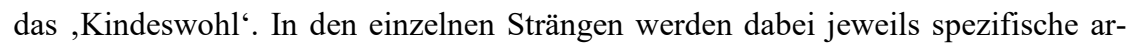
gumentative Figuren verwendet.

Unterschiede zwischen den Medien zeigen sich u.a. in der Beschreibung der politischen Gegner_innen und - immer damit verbunden - der Frage nach Adressat_innen und Zielgruppen. So ist es erklärtes Ziel und Inhalt der von uns untersuchten christlichen Blogs, Liberalisierungen innerhalb der Kirche etwa in Bezug auf die Anerkennung gleichgeschlechtlicher Partnerschaften anzuprangern. Segnungen eingetragener Lebenspartnerschaften dienen ebenso zur Empörung wie die Nachricht über eine neue kirchenrechtliche Regelung, die es gleichgeschlechtlichen Paaren gestattet gemeinsam im Pfarrhaus zu leben. Während kath.net und Charismatismus dies an die hierfür Verantwortlichen innerhalb der Kirche und an rechtskonservative Kirchenmitglieder adressieren, dient eine solche Meldung in der Jungen Freiheit maximal als vermeintlicher Beweis für das Ausmaß eines allgemeinen Verlustes von (christlichen) Werten.

Auch die Frage danach, ob gleichgeschlechtliche Partnerschaften per se abzulehnen sind oder es ,nur' darum geht, nicht-heterosexuellen Verbindungen eine andere Rechtsform und damit ungleiche Rechte gegenüber heterosexuellen Ehen zuzugestehen, wird nicht einheitlich beantwortet. In einzelnen Beiträgen christlichrechter Medien wird Homosexualität als „krank“ bezeichnet (Bauer 2013, in Charismatismus vom 10.06.2013). Insgesamt jedoch dominiert ein anderes, sicherlich in Teilen strategisch genutztes Narrativ: Gleichgeschlechtliche Partnerschaften unter dem Vorzeichen der Verbesonderung zu tolerieren, jedoch deren Sichtbarkeit und der rechtlichen Angleichung entgegen zu treten unter der Prämisse des Eheprivilegs für verschiedengeschlechtliche Paare.

„Gleichgeschlechtliche Partnerschaften sind keine Varianten der Ehe, auch nicht gleichwertige Alternativen. Sie sind das explizite Gegenteil! Sie sind hinnehmbar, wenn sie als Ausnahme erkennbar bleiben.“ (Baschang 2011, in kath.net vom 03.03.2011)

Als „hinnehmbar“, nicht aber gleichwertig anerkennenswert bezeichnet der Oberkirchenrat i.R. Klaus Baschang im März 2011 gleichgeschlechtliche Lebenspartnerschaften - und besteht dabei auf einer grundlegenden Verschiedenheit hetero- und homosexueller Verbindungen. Nicht die Unsichtbarmachung, sondern die permanente Betonung und Sichtbarmachung der Differenz steht im Kern der Argumenta- 
tion. Die Behauptung der grundlegenden Verschiedenheit von hetero- und homosexueller Partnerschaft sowie die Verschiedengeschlechtlichkeit als Grundlage von Ehe und Familie zieht sich durch sämtliche der nun folgenden Diskursstränge.

\subsection{Familie als verschiedengeschlechtliche Gemeinschaft}

Die Frage der Eheöffnung wird neben der Verschiedengeschlechtlichkeit der Eltern in erster Linie an die Frage geknüpft, wer dann ,Familie‘ sein kann. Familie wird heteronormativ verstanden als Form des Zusammenlebens heterosexueller Paare mit Kindern. In dieser Deutung werden verschiedengeschlechtliche Partnerschaften von anderen Formen des Zusammenlebens (Patchwork-Konstellationen, schwul-lesbische Partnerschaften ohne Kinder oder Regenbogenfamilien) abgegrenzt. Obwohl gerade das Zusammenleben von LGBTIQ* mit Kindern diese Gemeinschaften in ihrem Selbstverständnis zu Regenbogenfamilien macht, wird ihnen dieser Status abgesprochen:

„Ich habe auch Verständnis dafür, dass sich gleichgeschlechtliche Paare Kinder wünschen. Aber sie können aus sich heraus zusammen keine Familie gründen. Es ist somit legitim, einer solchen Verbindung einen anderen Namen zu geben." (Heber 2017, in kath.net vom 28.06.2017)

Hier werden gleichgeschlechtliche Elternpaare explizit nicht delegitimiert oder gar pathologisiert - aber deren Verschiedenheit zu heterosexuellen Elternpaaren betont. Beständig wird wiederholt, dass eine Verbindung zwischen zwei Eltern gleichen Geschlechts einen anderen Namen benötigt. An anderer Stelle dagegen wird gleichgeschlechtliche Elternschaft dezidiert in Frage gestellt:

„Das Wort ,Familie‘ ist, wie die Wörter ,Ehe‘, ,Eltern“ und ,verheiratet‘, schon jetzt zu einem unbrauchbaren Dunkelwort verkommen, bei dem man nicht mehr weiß, wovon eigentlich die Rede ist. Die Medien, die es sich leisten können, lügen sogar bewusst, um den Eindruck zu erzeugen, es gäbe so etwas wie natürliche gleichgeschlechtliche Eltern“ (Lombard 2013, in Freie Welt vom 02.07.2013).

Diese Deutung von Familie als exklusiver Verbindung zwischen Mann und Frau mit Kindern wird mit vermeintlich historischer Bedeutung aufgeladen. „Bis zum Ende der neunziger Jahre war für die Bürgerlichen eine Ehe die, grundsätzlich lebenslange, Verbindung von Mann und Frau zu dem Zweck, eine Familie zu gründen“, so Karlheinz Weißmann in der Jungen Freiheit (Weißmann 2015, in Junge Freiheit vom 12.06.2015). In ihrer Berichterstattung zur französischen Manif pour tous nimmt die Junge Freiheit vorweg, was sich ähnlich in den Argumentationen 
gegen die Ehe für alle wiederfindet: „Wichtig war den Veranstaltern dabei der Hinweis, daß sie keinesfalls als homophob verstanden werden wollen. Es ginge vor allem darum, auch weiterhin sicherzustellen, daß Kinder in Frankreich entsprechend den biologischen Realitäten einen Vater und eine Mutter haben", so Friedrich-Thorsten Müller, heutiger AfD-Politiker, in seinem 2012 veröffentlichten Beitrag Ein Papa, eine Mama, ganz einfach! (Müller 2012, in Junge Freiheit vom 23.11.2012; Schreibweise im Original).

Die politischen Auseinandersetzungen rund um die vollständige Öffnung der Ehe für gleichgeschlechtliche Partnerschaften in Frankreich waren für Teile der von uns untersuchten Akteure ein initialer Moment. Rekurse auf Frankreich sowie der Einbezug französischer Stimmen finden sich dementsprechend häufig. Die Freie Welt eignet sich in der Übersetzung des französischen Philosophen Bertrand Vergely dessen Argumentation eines Gegensatzes von Natur und Kultur an, indem sie von einer ,juristisch-medizinische[n] Bastelarbeit“ schreibt, die „Familie zu nennen [...] grober Unfug“" sei (Vergely 2013, in Freie Welt vom 23.04.2013).

Mit der Sozialwissenschaftlerin Gisela Notz lassen sich die hier gezeigten argumentativen Muster als familistisch beschreiben (Notz 2015). Notz kennzeichnet den Familismus als „Ideologie und den Anspruch der Exklusivität einer Familienform, die angeblich ,naturgegeben' und für die soziale Existenz jedes Menschen von zentraler Bedeutung ist" (Notz 2015: 8). Die Dichte an familistischen Argumentationsmustern in Debattenbeiträgen gegen die Ehe für alle bestätigt Notz' These, dass „der Familismus ein Comeback als konservative, auf den Wert der heterosexuellen, monogamen (Klein-) Familie bauende Politik [feiert]“ (ebd). Kern des Familismus ist die Bedeutungsaufladung der Familie als kleinster Zelle des Gemeinwesens. Darüber hinaus schließt der Familismus in der von Notz beschriebenen Form jede Vielfalt familialer Lebensweisen aus. Denkbar ist jedoch, dass sich familistische Argumentationen auch mit Formen vielfältigen familialen Zusammenlebens im Zuge der neoliberalen Aneignung geschlechtlicher und sexueller Lebensweisen verbinden lassen (Lang 2019) - findet sich die Vorstellung der Familie als Kern des Gemeinwesens schließlich auch in unterschiedlichen Wohlfahrtsregimes.

Das Anliegen Familie als verschiedengeschlechtlich zu definieren, reagiert auf die zunehmende öffentliche Sichtbarkeit von Regenbogenfamilien und die Tatsache, dass seit den 1990er Jahren zunehmend mehr Kinder in Familien mit gleichgeschlechtlichen Elternpaaren aufwachsen. Schwul-lesbische Organisationen beziehen sich dabei auf den widersprüchlichen Charakter der Ehe als Versorgungs- wie Disziplinierungs- bzw. Herrschaftsinstanz. Die Disziplinierungs- und Herrschaftsfunktion der Ehe erfuhren gleichgeschlechtliche Paare ex negativo - durch den nur langsam und schrittweise überwundenen Ausschluss von sozialen und kindbezogenen Rechten (Interview I). Zugleich rückte der Versorgungscharakter der Ehe stärker in den Vordergrund, als die gestiegene Zahl von Kindern in gleichgeschlechtlichen Partnerschaften neue Fragen aufwarf. Auf die daraus resultierenden rechtlichen Re- 
formen reagiert das von uns untersuchte politische Projekt mit dem Versuch, den Begriff der Familie wieder heteronormativ auf das Zusammenleben verschiedengeschlechtlicher Eltern mit leiblichen Kindern zu beschränken.

\subsection{Familie als Ort der Reproduktion}

Mit ihrer Verteidigung der heterosexuellen Kleinfamilie als einzig legitimem Ort, Kinder zu erziehen, verweisen die Auseinandersetzungen nicht zuletzt auf eine (Neu-)Aushandlung von Fragen sozialer und generativer Reproduktion. Die Legitimität des Zusammenlebens von Erwachsenen mit Kindern wird an die (zumindest potenziell mögliche) biologische Elternschaft geknüpft - die soziale Elternschaft in unterschiedlichen Formen familialen Zusammenlebens dagegen negiert. Damit fällt der hier propagierte Familienbegriff hinter das im Zuge der sozialliberalen Reformen der späten 1970er Jahre und auch von der heutigen Bundesregierung vertretene Familienverständnis zurück: Familie ist dort, wo Kinder sind. ${ }^{14}$ „Der wesentliche Unterschied zwischen gleichgeschlechtlicher Lebenspartnerschaft und Ehe besteht darin, daß ersterer die Potentialität für die Zeugung und Erziehung von Nachwuchs fehlt", so das damalige CDU-Mitglied Manfred Spieker anlässlich des irischen Referendums zur Einführung der gleichgeschlechtlichen Ehe (Spieker 2015, in Junge Freiheit vom 24.07.2015). ${ }^{15}$ Ehe und Familie werden damit nicht an das Zusammenleben mit, sondern in biologistischer Weise an die Zeugung von Kindern gebunden. Voraussetzung hierfür sei die Verschiedengeschlechtlichkeit der EhepartnerInnen. So heißt es in einem Beitrag der Freien Welt:

„Die Ehe kann nicht, geöffnet‘ werden, weil es gerade das Wesen der Ehe ist, lebenslanger Bund zu sein zwischen einem Mann und einer Frau, um gemeinsam Kinder zu zeugen und aufzuziehen - eine Familie zu gründen. Das gilt auch dann, wenn die Absicht des Kinderbekommens sich nicht erfüllt.“ (Krause 2015, in Freie Welt vom 12.06.2015)

Weder die individuelle Entscheidung für oder gegen ein Zusammenleben mit Kindern, noch das tatsächliche Zusammenleben mit (eigenen) Kindern gerät hier zum Kriterium für Familie oder das Rechtsinstitut der Ehe - sondern alleinig die angenommene „Absicht des Kinderbekommens“. So unterstellt Ulrich Kutschera: „Mit der faktischen Abwertung potenziell fruchtbarer Ehen, gemäß dem genderistischen Gleichstellungs-Glauben, wird die Geburtenrate vermutlich eher abfallen als anstei-

14 Vgl. exempl. das Urteil des Bundesverfassungsgerichts zur Unterhaltspflicht nichtverheirateter Elternpaare (BVerfG, Beschluss des Ersten Senats vom 28. Februar 2007 1 BvL 9/04).

15 Spieker verließ später aus Protest gegen die Entscheidung zur Ehe für alle die CDU. 
gen.“ (Kutschera 2017b, in kath.net vom 18.09.2017;16 vgl. auch Näser-Lather in diesem Band).

Aus der ,Ehe als Reproduktionsgemeinschaft" abgeleitet wird eine vermeintlich ,natürliche" Ungleichwertigkeit familialer Lebensweisen, die eine rechtliche Ungleichbehandlung rechtfertigen. Das beinhaltet ein

„Verständnis von ,Ehe“ als dauerhafter Gemeinschaft von Mann und Frau, offen auf Nachkommenschaft und als einziger Ort, an dem Menschen auf natürliche Weise gezeugt und zur Welt gebracht werden.“ (Küble 2017, in Charismatismus vom 30.06.2017)

Es wird ein Gegensatz von ,Natur' versus ,Kultur' beschrieben, in denen die ,Natur' zum unhinterfragbaren Kontinuum erklärt wird. ,Kultur` dagegen gilt als künstlich geschaffen und deswegen abwendbar. Die Formulierungen zeigen Parallelen zum auf Reproduktion fixierten Diskurs selbsternannter ,Lebensschützer' (Sanders/ Jentsch/Hansen 2014). Als „lebensfeindlich“ beschrieben wird hier, was nicht die biologische Reproduktion der Gemeinschaft als primäres Ziel zwischenmenschlicher Beziehungen absolut setzt. Einzelne Akteure gehen noch weiter und ordnen die Fixierung auf den Bereich der Reproduktion ein in ein demografisches Krisenszenario. So wird in neurechten Medien, die sich wie oben beschrieben explizit zwischen konservativ-rechten und neonazistischen Positionen verorten, eine Verbindung gezogen zwischen demografischer Entwicklung und der Öffnung der Ehe (vgl. auch: Kutschera 2017b, s.o.):

„Ich muss nicht den Katechismus der katholischen Kirche zum Maßstab machen, um zu erkennen, dass in unserer Gesellschaft einiges schiefläuft, von der steigenden Scheidungsrate (,Liebes-Aus') bis dahin, dass Deutschland die niedrigste Geburtenrate der Welt hat. Für wessen Emanzipation kämpfen wir eigentlich, wenn wir selber darüber aussterben?“ (Lombard 2015, in Freie Welt vom 03.12.2015)

Der neurechte Intellektuelle Karlheinz Weißmann nimmt in seinem Beitrag für die Junge Freiheit gar Bezug auf das rechte Angstphantasma vom „Volkstod“ (Weißmann 2015, in Junge Freiheit vom 12.06.2015; vgl. auch Botsch/Kopke 2019). Dabei werden wie in dem Beitrag von Fischer Fragen individueller und gesellschaftlicher Reproduktion verknüpft:

16 Das hier zitierte Interview ist Gegenstand eines juristischen Verfahrens gegen Ulrich Kutschera, Kutschera muss sich u.a. wegen des Verdachts der Volksverhetzung, Beleidigung und Verleumdung vor Gericht verantworten (Stand November 2019). 
„EHE beinhaltet nicht nur die , auf Dauer angelegte, rechtlich verfestigte Partnerschaft' zwischen zwei Menschen, sondern auch die Verantwortung für den Fortbestand der demografischen, der sozialen und der kulturellen Gesellschaft. Ein Staatswesen, das nicht mehr auf Kontinuität setzt, hat bereits verloren.“ (Fischer 2015a, in Freie Welt vom 10.06.2015, Herv. i.O.)

Der Kontroll- und Disziplinierungscharakter der untersuchten Positionen im politischen Projekt wird besonders deutlich an deren Implikationen für das Themenfeld der sozialen und generativen Reproduktion. Ehe und Familie werden gleichgesetzt und als heterosexuelle und verschiedengeschlechtliche Reproduktionsgemeinschaft verstanden. Beide seien dem demografischen Erhalt des Staatsvolkes verpflichtet. Ein solches Verständnis definiert zugleich, welche Mitglieder der Gesellschaft sich nicht an deren generativen Reproduktion beteiligen sollen: alle nicht eindeutig heterosexuellen und (im erweiterten Sinne) monogam lebenden Menschen. Nicht zufällig finden sich dabei explizit völkische Argumentationen, in denen die Generationenfolge als Kriterium gegen die Ehe für alle verwendet wird, so etwa wenn es heißt, ,daß in einer gleichgeschlechtlichen Verbindung die Generationenfolge nicht möglich ist." (Geis 2017, in Junge Freiheit vom 07.07.2017). Es wird hier auf eine Blutslinie und damit auf ein dezidiert völkisches Verständnis einer (Abstammungs-) Gemeinschaft abgehoben. Dies ist mit ordnungspolitischen Entwürfen verknüpft, die das Individuum nur als Träger_innen einer ihm oder ihr angetragenen Rolle sehen.

Der Kontroll- und Disziplinierungscharakter zeigt sich ebenfalls in der Frage, welche Gesellschaftsmitglieder die anfallenden Reproduktionsarbeiten verrichten sollen. Ungeachtet der vorliegenden Erkenntnisse über die damit verbundenen Prekarisierungsrisiken (Klenner et al. 2011) wird im untersuchten Material weiterhin die traditionelle heterosexuelle Kleinfamilie mit männlichem Familienernährer und weiblicher Hausfrau propagiert. Dass innerhalb dieser familistischen Strukturen (Notz 2015) eine strukturelle Ungleichverteilung von Care-Arbeit vorliegt, da diese Aufgaben den Frauen als natürlicher Tätigkeitsbereich zugeschrieben werden, bleibt dabei unerwähnt. Verdeckt wird auch, dass rund um die in den Texten häufig als isoliertes Atom diskutierte Familie ein komplexes Netz an zusätzlichen Reproduktionsstrukturen wie etwa Kitas, häusliche Pflege oder Altersheime existiert, in welches ursprünglich zur Familie gehörige Pflegeaufgaben ausgelagert sind. Gerade in diesen Arbeitsverhältnissen wird jedoch verstärkt auf prekarisierte Arbeitskräfte (Migrant_innen oder Geringverdiener_innen) und irreguläre Arbeitsverhältnisse zurückgegriffen. Die rassistischen und sozialdarwinistischen Implikationen dieser Entwicklung sollen innerhalb der nationalistisch gedachten Reproduktionsstrukturen auf Dauer gestellt und soziale Ungleichheit festgeschrieben werden. 


\subsection{Verhältnis zum Staat: Die „Familie als Grundlage des Gemeinwohls“"17}

Der Ehe kommt im von uns untersuchten Material die mehrfache Funktion zu, nicht nur die Nachkommenschaft zu sichern, sondern auch zwischenmenschliche Beziehungen im Allgemeinen zu ordnen. Die Organisation der sozialen Reproduktion und die Entfaltung der Sexualität bedürften demnach der Kontrolle und Disziplinierung. ${ }^{18}$ „Den Ehe-Begriff uferlos auszudehnen und daraus gleiche Rechte für die unterschiedlichsten Akteure abzuleiten heißt, die Keimzelle der Gesellschaft anzugreifen“, so Thorsten Hinz in der Jungen Freiheit (Hinz 2015, in Junge Freiheit vom 12.06.2015). Die von uns untersuchten Medien offenbaren insbesondere in diesem Punkt jeweils spezifische Auffassungen zum Verhältnis von Staat, Gesellschaft und Politik. Einige AutorInnen im christlich-rechten Spektrum nehmen Bezug auf die katholische Soziallehre mit ihren Aussagen zu Ehe und Familie.

„Die Ehe zwischen Mann und Frau ist die Grundlage der Familie. Die Familie ist wiederum die Grundlage der Gesellschaft. Die katholische Soziallehre bezeichnet sie als die ,Keimzelle der Gesellschaft‘. Dies bedeutet, dass die Ehe nicht nur eine immens hohe zivilisatorische Bedeutung besitzt, sondern auch (historisch und philosophisch) vor dem Staat existiert. Es steht deshalb dem Staat nicht zu, den Begriff der Ehe umzudefinieren.“ (von Gersdorff 2017, in Charismatismus vom 29.06.2017)

Die Ehe wird hier als dem Staat übergeordnete Institution benannt. Dies steht im Widerspruch zu rechts-konservativen und neurechten Stimmen, die an den Staat appellieren, die Institution der Ehe zu ,schützen“ - sie also wie gehabt in heteronormativer Weise zu regulieren. Begründet wird dies mit der Funktion von Ehe und Familie für Staat und Gesellschaft.

„Aus soziologischer Sicht haben Ehe und Familie deshalb gesellschaftliche Funktionen. Aus ökonomischer Sicht produzieren sie positive externe Effekte. Diese vitalen Funktionen von Ehe und Familie verbieten es, gleichgeschlechtlichen Partnerschaften ehegleiche Rechte ein-

17 Kuby 2013, in kath.net vom 18.01.2013.

18 Die Fixierung auf reproduktive Sexualität im Reden über gleichgeschlechtliche Paare offenbart auch der Evolutionsbiologe Ulrich Kutschera, wenn er im Interview mit kath.net über „,[g]leichgeschlechtliche Erotik-Akte ohne das Potenzial zur Kinderproduktion“ spricht, „die man auch (wertfrei) als biologisch sinnloses Paarungs-Verhalten bezeichnen“ könne. Diese, so Kutschera, ,sind nicht gleichzustellen mit Mann-Frau-Verbindungen. Diese können im Prinzip eine ,fruchtbare Ehe“ führen“ (Kutschera 2017b, in kath.net vom 18.09.2017). 
zuräumen. Eingetragene Lebenspartnerschaften leisten zur Regeneration der Gesellschaft keinen Beitrag. Sie haben weder positive externe Effekte noch gesellschaftliche Funktionen.“ (Spieker 2015, in Junge Freiheit vom 24.07.2015)

Das Blog Charismatismus, an anderer Stelle auf den christlichen Wert der Nächstenliebe erpicht, rückt die Frage der Reproduktion des Staatsvolkes in den Mittelpunkt eines neoliberalen Kosten-Nutzen-Denkens. Das nachfolgende Zitat bezieht sich auf die damalige CDU-Politikerin Erika Steinbach:

„Frau Steinbach [...] stellte gleich eingangs ihre Position klar: ,Jeder kann leben, wie er möchte, aber der Staat muß nicht alles finanziell fördern, sondern speziell das, was seiner $\mathrm{Zu}-$ kunft dient ${ }^{\star}$ - und das sei die herkömmliche Ehe mit natürlicher Nachkommenschaft. Das ist tatsächlich ein logisch zu Ende gedachtes, einleuchtendes Prinzip.“ (Küble 2013, in Charismatismus vom 13.06.2013)

Ehe und Familie werden auf die Funktion der Fortpflanzung reduziert und staatliche Anerkennung an das potenzielle Vorhandensein von Kindern gebunden. Dies ist das Gegenteil des durch Matthias von Gersdorff angebrachten Arguments, die Ehe als , vorstaatlich“ zu beschreiben. Hier wird staatliches Handeln explizit angerufen zur Verteidigung heteronormativer familialer Lebensweisen. Diese argumentativen Muster mischen sich mit einem rechtspopulistischen Anti-Etatismus (siehe unten).

Die Vorstellung der Familie als nutzenorientierte kleinste Zelle des Gemeinwesens bei gleichzeitiger Indienstnahme der Familie für Gesellschaft und Staat ist nicht neu. Der Soziologe Andreas Kemper verweist auf dessen nationale Spezifik:

„Das Narrativ Familie als Keimzelle der Nation scheint vor allem im organizistischen Staatsverständnis Deutschlands und Österreichs eine wichtige Rolle zu spielen. Im Englischen ist dieses Narrativ quasi nicht vorhanden, dort wird weniger biologistisch von der Familie als ,Foundation of the Nation“, als ,Fundament der Nation“ gesprochen.“ (Diskursatlas 2019, Herv. i.O.)

Biologismus wie auch Familismus finden sich also in der behaupteten Naturhaftigkeit der heterosexuellen Ehe sowie deren Nutzen für Staat und Gemeinschaft. Dabei widersprechen sich die vorgefundenen Positionen in ihren Appellen an staatliches Handeln bzw. Nicht-Handeln. Familistische Vorstellungen lassen sich hier mit unterschiedlichen Vorstellungen von Staat und Staatlichkeit verbinden. Im Kern der Argumentation steht die Familie als kleinste Zelle des Gemeinwesens. Wie dieses konkret ausgestaltet ist und welche Rolle staatlichem Handeln zur Verteidigung normativer Familienformen zukommt, wird verschieden beantwortet. 


\section{4 „Kindeswohl“}

Auf einer anderen Ebene bewegen sich der Diskursstrang des ,Kindeswohls' ${ }^{`}$ und die normativ aufgeladene Frage nach dem Schutz von Kindern. Hier wird Deutungswissen über Themen rund um Geschlecht, Sexualität und familiale Lebensweisen geteilt, die nur peripher etwas mit der Verabschiedung der Ehe für alle zu tun haben - aber den Rückgriff auf die normativ aufgeladene Anrufung des ,unschuldigen Kindes" (Schmincke 2015) erlauben.

In den argumentativen Mustern rund um das Thema ,Kindeswohl' wird eine grundsätzliche Schlechterstellung bis Gefährdung von Kindern in Regenbogenfamilien bzw. durch die Ehe für alle behauptet. ${ }^{19}$ Wir finden hier eine Kongruenz mit argumentativen Mustern aus dem parlamentarischen Prozess und den christlichrechten und neurechten Medien aus unserem Korpus.

Die argumentative Figur der Verschiedengeschlechtlichkeit der Eltern wird auch hier genutzt und zum Kriterium für das Wohlergehen von Kindern erklärt.

„Kinder sind eigene Personen und haben Anspruch auf den Schutz ihrer Würde und ihrer Entfaltungsmöglichkeiten. Die Optimierung dieser Entfaltungsmöglichkeiten erfordert Erzieher beiderlei Geschlechts.“ (Spieker 2015, in Junge Freiheit vom 24.07.2015)

Mit Verweis auf eine wiederholt zitierte Studie aus Kanada wird behauptet, Kinder vor drohendem Leid schützen zu müssen - vor ,signifikant nachteilige[n] Ergebnisse[n] (im Hinblick auf Bildungs- und Berufserfolg, Gesundheit, Delinquenz, Drogenmissbrauch, Suizidgefährdung, etc.)“ (Cornides 2013, in kath.net vom 27.05. 2013). Der Bezug auf diese wissenschaftlich längst widerlegte Studie vermittelt den Anschein von Objektivität und Faktizität - und lässt sich als argumentative Strategie beschreiben mit dem Ziel, der eigenen Position Gewicht zu verschaffen. Eine solche Argumentation verläuft nicht widerspruchsfrei und eignet sich argumentative Figuren der Befürworter_innen der Ehe für alle an, so etwa die der Selbstbestimmung von Individuen.

„Mit der Homo-Ehe, die das Recht auf künstliche Befruchtung einschließt, wird genau dies verdreht und das Recht des Kindes dem Recht auf Kinder geopfert. Unter dem Vorwand, Homosexuellen ein Recht auf Kinder geben zu wollen, wird das Kind von der Person zum Objekt degradiert. Während Menschenrechtsvertreter in aller Welt gegen die Verdinglichung

19 In zwei Beiträgen im Korpus wird die Homo-Ehe in Verbindung gerückt mit Pädophilie und Kindesmissbrauch, beide stammen vom Ulrich Kutschera (Kutschera 2017a, b; kath.net). 
des Menschen kämpfen, wird das Kind im Namen des Rechts der Homosexuellen zum bloßen Objekt.“ (Vergely 2013, in Freie Welt vom 23.04.2013)

Besonders hervorgehoben wird das mit der Eheschließung verbundene Recht, Kinder zu adoptieren. Dies war, wie bereits erwähnt, auch in Frankreich der Anstoß zur Mobilisierung gegen die Eheöffnung.

„Das gilt besonders für das volle Adoptionsrecht. Hier sehe ich das Kindeswohl beeinträchtigt. Ich bin davon überzeugt, dass die Bipolarität der Geschlechter von Mann und Frau, die ja erst menschliches Leben zeugt und ohne die kein Kind gezeugt werden kann, auch für die Erziehung und Formung von Kindern nach ihrer Geburt gut und notwendig ist.“ (Bischoff Fürst 2015, in kath.net vom 22.06.2015)

Die Geschlechter- und Sozialwissenschaftlerin Imke Schmincke hebt in ihrem Vergleich deutscher und französischer Mobilisierungen hervor, dass in beiden Ländern die Öffnung der Ehe von rechter und konservativer Seite frühzeitig als Gefährdung des Wohles von Kindern kritisiert und abgelehnt wurde. Eine (vorgebliche) Akzeptanz homosexueller Lebensweisen wird der Nicht-Akzeptanz homosexueller Elternschaft gegenübergestellt - unter Bezugnahme auf das Wohl von Kindern. Neben der vermeintlich ,unschlagbare[n] Macht, [den] die argumentative Referenz auf die Kinder und mehr noch das Kindeswohl hat“, welche ,die eigene Position stärkt und alternative Positionierungen zum Verstummen bringt“" (Schmincke 2015: 93), verweist dies auf eine lange Tradition:

„Die Referenz auf Kinder als moralischer Waffe hat [...] eine lange (und vor allem christliche!) Tradition, in die sich die neueren Inanspruchnahmen einschreiben können. Als Waffe dient sie dann jedoch ganz anderen Zwecken, wie der Profilierung, der Durchsetzung von Interessen oder der Legitimation sozialer Ungleichheit.“ (Schmincke 2015: 102)

Die Macht der argumentativen Figur des ,unschuldigen Kindes', das zu beschützen in der Hand von Staat und Gesellschaft liege, soll die moralische Überlegenheit, Authentizität und Glaubwürdigkeit der SprecherInnen bezeugen. Eben jene moralische Macht nutzen die TrägerInnen eines antifeministischen, regressiv-autoritären politischen Projektes, um ein spezifisch normatives Wissen um Geschlecht und Sexualität im Diskurs zu verankern.

Die Argumentation mit dem vermeintlichen ,Kindeswohl' bewegt sich dabei im Rahmen einer Verschiebung antifeministischer Argumentationen der vergangenen Jahrzehnte weg vom ,männerzentrierten“ Antifeminismus der 1990er und frühen 2000er Jahre hin zum „familienzentrierten“ Antifeminismus der jüngeren Zeit, in dem die Figur des ,unschuldigen Kindes` zentral gesetzt wird für die Ablehnung von Politiken der Vielfalt geschlechtlicher und sexueller Vielfalt (Scheele 2016). 
Die Knüpfung des ,Kindeswohls‘ an das Aufwachsen mit verschiedengeschlechtlichen Eltern greift zurück auf geschlechtsspezifisch vergebene Rollen an Männer und Frauen. Außerhalb der heterosexuellen Kleinfamilie mit ihrer traditionellen Rollenverteilung und Reproduktionsstruktur, so die Idee, könne es keine behütete Kindheit geben, es drohen vielmehr Identitätskonflikte und psychische Schäden. Dabei kennt Mütterlichkeit kein Geschlecht (Krüger-Kirn/Tichy in diesem Band). Der Bezug auf das ,Wohl des Kindes' verdeckt bewusst den (hetero-)normativen Charakter der hier aufgerufenen spezifischen Form familialen Zusammenlebens - samt der ihm innewohnenden ungleichen Geschlechterordnung.

\section{FORMIERUNG EINES AUTORITÄR-REGRESSIVEN ProjeKtS IM Kontext DER DEbatte UM DIE EHE FÜR ALLE}

Angesichts der strömungsübergreifenden Bezugnahme auf die von uns skizzierten Diskursstränge im Untersuchungszeitraum zwischen 2011 und 2017 zeigt sich die Formierung eines antifeministischen, autoritär-regressiven Projektes im Bereich der Geschlechterpolitik. Diese Formierung lässt sich sowohl in struktureller wie auch in diskursiver Hinsicht beobachten. In der Bewertung des politischen Projektes einerseits und zur Beantwortung der Frage nach der Formierung dessen andererseits gilt es demnach, beide Dimensionen zusammen zu denken.

\subsection{Strukturelle Dimension}

Im Fokus standen bislang die geteilten argumentativen Figuren und Diskursstränge. Nichtsdestotrotz zeigt ein Blick auf die AutorInnenschaft der untersuchten Medien deutliche Überschneidungen zwischen dem christlich-rechten und dem neurechten Spektrum unseres Korpus auf. Insbesondere die Mehrfachautorenschaft einzelner Akteure in unterschiedlichen Medien zeugt von einer Formierung als strömungsübergreifendem politischen Projekt, die über eine bloße Bezugnahme auf geteilte Argumentationsstränge und -figuren hinausgeht. Exemplarisch nennen wollen wir hier Matthias von Gersdorff, Andreas Lombard und Stefan Fuchs. Der katholische Publizist und Volkswirt Matthias von Gersdorff steht für den christlich-klerikalen Teil des politischen Projektes. Als Leiter der Deutschen Vereinigung für christliche Kultur e.V. (DVCK e.V.) argumentiert er nicht nur gegen die Eheöffnung, sondern wirkt auch als umtriebiger Netzwerker hinter der Aktion Kinder in Gefahr und lanciert Kampagnen gegen eine angebliche ,Gender-Ideologie‘ mit Bezug nach Hessen. Von Gersdorff veröffentlichte im Untersuchungszeitraum Beiträge sowohl im neurechten Blog Freie Welt als auch im christlich-rechten Blog Charismatismus. 
Darüber hinaus stand er der Jungen Freiheit als Gesprächspartner für ein Interview zur Verfügung. Er positioniert sich damit an der Schnittstelle zwischen unterschiedlichen Medien und deren jeweiligen Zielgruppen und vernetzt das politische Projekt in Persona. Ähnlich der Journalist und Verleger Andreas Lombard: Er gehört dem neurechten Teil des politischen Projektes an und veröffentlicht in erster Linie im Blog Freie Welt. Nichtsdestotrotz tauchte er im Untersuchungszeitraum ebenso als Autor auf dem katholisch-klerikalen Portal kath.net auf. Als Drittes zu nennen ist Stefan Fuchs. Fuchs ist wissenschaftlicher Mitarbeiter am Institut für Demographie, Allgemeinwohl und Familie (IDAF), einer Organisation, die als klerikal-konservative ,Ideenfabrik' über enge Verbindungen zur Konrad-Adenauer-Stiftung und zu Teilen der christlich-konservativen Rechten verfügt.

Die Autorenschaft der drei genannten Protagonisten zeigt stellvertretend deren personelle und diskursive Vernetzung. Es zeigt sich insbesondere am Beispiel von Matthias von Gersdorff, dass sich einzelne Akteure als Teil eines politischen Projektes verstehen, das den eigenen Aktivismus nicht auf das Verfassen von Beiträgen für christliche und neurechte Blogs und Zeitungen beschränkt. Alle drei von uns genannten Autoren engagier(t)en sich nicht nur in Gegnerschaft zur Ehe für alle, sondern beziehen auch anderenorts autoritär-regressive und im Kern antifeministische Positionen in Debatten rund um Geschlecht und Sexualität. In diesem Sinne zeigt sich hier ein Effekt, der sich in Anlehnung an Gessenharter (1990: 66f) als Scharnierfunktion des politischen Projektes beschreiben lässt - verschiedene politische Milieus verschränken sich miteinander, über personelle Schnittmengen wie über geteilte diskursive Bezüge.

\subsection{Diskursive Dimension}

Die gegenseitige Bezugnahme sowie die Mehrfachautorenschaft einzelner Protagonisten sprechen für eine strukturelle wie auch diskursive Nähe zwischen Neuer und christlicher Rechter. Im Kern der Gegnerschaft zur Ehe für alle steht eine geteilte Problemdefinition, die in unterschiedlichen Diskurssträngen verargumentiert wird: Die geplante Eheöffnung stelle die Grundlagen von Familie, Staat und Gesellschaft in Frage und gefährde das Wohlergehen künftiger Generationen.

Das politische Projekt formiert sich nicht nur im Bezug der Akteure aufeinander, sondern mindestens ebenso in der geteilten Abgrenzung zu einem übereinstimmend als , links-rot-grün' definierten Gegenüber. Graduelle Unterschiede in der Beschreibung jenes Gegenübers lassen sich aufzeigen: So verwendet das christliche Blog Charismatismus den Begriff mehrheitlich für tatsächlich unter Beteiligung von SPD und Grünen verabschiedete politische Maßnahmen - wie etwa das Lebenspartnerschaftsgesetz - und für Entwicklungen innerhalb der Kirche, die sie darauf zurückführen. Dagegen erfährt der Begriff eine weit darüberhinausgehende 
Aufladung, wenn etwa in der Jungen Freiheit die Rede ist von einer „einflußreichen Homosexuellen-Lobby [und den] eng verbundenen Grünrotlinke[n]“ (Paulwitz 2013, in Junge Freiheit vom 01.03.2013) oder gar von einem ,lupenreine[n] Kommunismus im rotgrünen Gewand“ (Fischer 2015b, in Freie Welt vom 16.10.2015). Die AutorInnen der neurechten Medien Junge Freiheit und Freie Welt verstehen die Auseinandersetzungen damit stärker als ihre MitstreiterInnen aus den christlichen Portalen als ,Kampf ums Ganze' und verleihen konkreten Schritten rechtlicher Anerkennung und Sichtbarmachung sexueller und geschlechtlicher Vielfalt eine weitergehende Bedeutung.

„Dieser Familie, die nach wie vor das Ideal der übergroßen Majorität im Volk ist, entzieht die von Karlsruhe mit vorangetriebene völlige Gleichstellung von homosexuellen Partnerschaften ihren besonderen Rang und liefert sie der Beliebigkeit und dem Relativismus aus. [...] Einmal eingerissen, sind Grenzen kaum mehr neu zu ziehen." (Paulwitz 2013, in Junge Freiheit vom 01.03.2013)

Insbesondere in den diskursiven Konturen des politischen Projektes sehen wir deutliche Überschneidungen zu Figurationen des zeitgenössischen ,Rechtspopulismus (Priester 2016), dessen Anliegen es ist, Grenzen der Nation bzw. der (Volks-) Gemeinschaft nach innen wie nach außen zu verteidigen. Geschlecht und Sexualität dienen hierbei als Ordnungskategorien für die eigene Gemeinschaft (Sauer 2017). Unter Rückgriff auf vermeintlich überhistorische, naturalisierte Geschlechter- und Sexualitätsdispositive wird nach einer „Naturalisierung sozialer Ungleichheit“ gestrebt (Mayer et al. 2018: 271). Ähnliches lässt sich an der Gegnerschaft zur Ehe für alle zeigen. Dies überrascht nicht, gehören Teile der analysierten Medien doch selbst zu jenen Echokammern, die sich zwischen den Polen der konservativen und der völkischen Rechten bewegt.

Jenseits der politischen Verortung einzelner AutorInnen finden sich in allen untersuchten Medien wiederholt argumentative Muster eines, Wir' versus, die Anderen'. Diesen ,Anderen' wird zugeschrieben, eine hegemoniale Position inne zu haben und durch die Umsetzung der Ehe für alle Geschlechter- und Sexualitätsverhältnisse in Unfrieden zu bringen. Dies weist deutliche Parallelen zum im Rechtspopulismus aufgemachten Konflikt zwischen ,dem Volk' und vermeintlich ,manipulativen Eliten“ auf, „,seien dies politische Eliten, ,das' Establishment, die sogenannte Lügenpresse, MigrantInnen, Schwule oder Feministinnen.“ (Sauer 2017: 2) Den ,Anderen' wird häufig ein Mehr an Macht - eine einflussreiche Lobby-Funktion - zugeschrieben und gleichsam angekreidet. So ist im Untersuchungszeitraum zur Ehe für alle gehäuft die Rede von einer ,Homo-Lobby', der angelastet wird, die Mehrheit zu dominieren. „Die Homosexuellen-Lobby ist winzig - aber einflußreich. In wenigen Jahren hat sie unsere Gesellschaft verändert. Was ist das Geheimnis ihrer 
Macht? Welche Strategien verfolgt sie?", kündigt ein Redakteur der Jungen Freiheit sein Interview mit dem Lebensschutz-Aktivisten Matthias von Gersdorff an (Schwarz 2011, in Junge Freiheit vom 14.01.2011). Und der neurechte Autor Michael Paulwitz sieht schon 2009 in der Entscheidung der Bayerischen Landesregierung, ihre Klage gegen das Lebenspartnerschaftsgesetz zurück zu ziehen, einen

„,machtvolle[n] Beweis für das Gewicht der Homosexuellen-Lobby in der politischen Landschaft. Mit dem Lesben- und Schwulenverband Deutschlands (LSVD) als Kristallisationspunkt erntet sie derzeit die Früchte eines jahrzehntelangen Ringens um kulturelle Hegemonie. [...] Der Verband, 1990 als Schwulenverband in der DDR gegründet und 1999 in LSVD umbenannt, ist ein Lehrbeispiel dafür, wie eine im vorpolitischen, medialen, gesellschaftlichen und medizinischen Bereich gedeihende Ideologie durch geschickte Vernetzung und aggressive Lobbyarbeit auf allen Ebenen in politischen Einfluß umgemünzt wurde.“ (Paulwitz 2009, in Junge Freiheit vom 28.08.2009)

Paulwitz schreibt dies im Jahre 2009, also zu einem Zeitpunkt, der vor unserem Untersuchungszeitraum liegt. Er formuliert hier Anwürfe gegen eine angebliche „Homosexuellen-Lobby“, die uns auch im Untersuchungszeitraum begegnen - und den Terminus der ,Homo-Lobby` zu einer eigenen argumentativen Figur werden lassen. Diese Figur zeigt deutliche Parallelen zu anti-etatistischen Argumentationsmustern rechtspopulistischer Akteure heute, zehn Jahre später. Schmincke kennzeichnet diese Form des Anti-Etatismus als eine Spezifik der deutschen Gegnerschaft zur Eheöffnung: Während bei den französischen GegnerInnen der Homo-Ehe eher ein universalistischer Diskurs vorherrsche, werde in Deutschland stärker mit Figuren der „Umerziehung', ,Lobbygruppen“ etc. argumentiert (Schmincke 2015: 100).

Mit seiner Benennung von Feldern, in denen der LSVD versuche eine kulturelle Hegemonie zu erringen, nimmt Paulwitz zudem die Felder autoritär-regressiver Mobilisierungen der Folgejahre vorweg. Insbesondere im vorpolitischen und medialen Bereich ringen rechts-autoritäre Akteure heute ungleich aggressiver als noch vor zehn Jahren um Einfluss und versuchen eigenes Deutungswissen zu verankern nicht nur, aber unter anderem um Geschlecht und Sexualität.

Wie ambivalent dabei die vermeintlichen Beweisführungen sind, zeugt der widersprüchliche Bezug auf die argumentative Figur von ,Mehrheit versus Minderheit': So wird im Untersuchungszeitraum die Verabschiedung der Ehe für alle durchgängig als Auseinandersetzung zwischen Mehrheit und Minderheit interpretiert. Dabei finden wir zum einen Äußerungen, die sich in der Reklamierung der eigenen, heteronormativen Position als Mehrheitsposition gegen die Eheöffnung wenden. Wir finden ebenso Beiträge, welche die Argumentationsfigur umdrehen und sich selbst als Minderheit gegenüber einer Mehrheit der Befürworter_innen sehen und auf dieser Grundlage einen Minderheitenschutz fordern. Es handelt sich hier um eine gezielte Vereinnahmung des Begriffes der Diskriminierung und damit ver- 
wandter Termini aus dem Bereich des Minderheitenschutzes, die je nach Argumentationszusammenhang mit unterschiedlichen Bedeutungen aufgeladen werden. Ziel bleibt es, die eigene Position zu legitimieren. Dass dieses Ringen um die Definitionsmacht über entscheidende Begriffe die Debatten um die Eheöffnung insgesamt begleitet hat, schilderten auch involvierte Beobachter_innen des politischen Prozesses (exemplarisch Interview I).

Hier wurden sich Argumente der Befürworter_innen der Ehe für alle angeeignet und ihres ursprünglichen Gehaltes entleert. Der Bezug auf einen von uns ausgemachten diskursiven Strang, der sich rund um die widersprüchliche Vereinnahmung von Begriffen wie Diskriminierung oder des Minderheitenschutzes gruppiert, ist dabei als eine diskursive Strategie zu verstehen, die langfristig unter anderem darauf zielt, dem autoritär-regressivem Projekt Einfluss und Handlungsspielräume in „der sogenannten bürgerlichen Mitte“ zu verschaffen (Schutzbach 2018: 27). Wie eingangs ausgeführt, werden diskursive Transformationen und Neukonfigurationen von Stützungsverhältnissen durch neue diskursive Muster begleitet. Dies trifft auch $\mathrm{zu}$ auf den hier beschriebenen Versuch einer Neufiguration hegemonialer Geschlechter- und Sexualitätsverhältnisse in der Moderne.

\section{FAZIT UND Ausblick: IMPLIKATIONEN}

Das von uns untersuchte antifeministische, autoritär-regressive Projekt konnte die Einführung der Ehe für alle nicht verhindern und ist daher im strengen Sinne gescheitert. Allen Versuchen zum Trotz, ein heteronormatives Wissen um Geschlecht, Sexualität und die eigene Deutung von Familie hegemonial im Diskurs um die Ehe für alle zu verankern, verabschiedeten Bundestag und Bundesrat im Sommer 2017 das Gesetz zur gleichgeschlechtlichen Eheschließung. Am 01. Oktober 2017 erfolgten die ersten Eheschließungen.

Der politische Prozess um die Ehe für alle diente Teilen ihrer GegnerInnen als Anlass der Formierung eines politischen Projekts - weitere Anlässe werden folgen. Darauf deutet die selbstbewusste Ausrufung eines ,Kulturkampfs ' durch die von uns untersuchten Akteure hin. Für die zukünftige Entwicklung dieser Konflikte um Hegemonie wird es entscheidend sein, welche gesellschaftliche Anschlussfähigkeit das antifeministische, autoritär-regressive Projekt weiterhin entwickeln und inwieweit es eine Scharnierfunktion für verschiedene soziale und politische Spektren einnehmen kann. Im Bereich der Geschlechter- und Sexualitätsverhältnisse sehen wir fortbestehende Resonanzräume für Argumentationen gegen die tatsächliche Gleichstellung und Sichtbarkeit homosexueller Lebensweisen. Während die Akzeptanz nicht-heterosexueller Lebensformen seit der Jahrtausendwende generell zunimmt und die Ehe für alle Zustimmungswerte von bis zu 83 Prozent in Umfragen 
erreichte, existieren weiterhin verdeckte Formen von Homophobie (Dernbach 2017; Kram 2018). Auch der in allen Teilen der Gesellschaft verbreitete Familismus bietet weiterhin ein Einfallstor. Hier knüpft das autoritär-regressive Projekt mit seinem Bezug auf die heteronormative Kleinfamilie inhaltlich flexibel an gesellschaftliche Debatten und andere politische Projekte an - immer mit dem Ziel, Zustimmung und damit Mehrheiten im, aber auch jenseits des rechts-konservativen Lagers zu gewinnen.

Über den konkreten Kontext der Debatten um die Ehe für alle eröffnete sich ein Möglichkeitsraum für ein antifeministisches, autoritär-regressives Projekt, welches sich über den konkreten Anlass hinaus formiert - und anlassübergreifend für eindeutige, heteronormative Geschlechterrollen eintritt. Dies scheint der einende Bezugspunkt in einem größer angelegten Kampf um Hegemonie im Bereich der Geschlechter- und Sexualitätsverhältnisse zu sein. Wo im autoritär-regressiven Verständnis eine ,stabile, eindeutige, ursprüngliche Identität [...] als gefährdet“ (Wielowiejski 2018: 354) erscheint, entstehen Abwehrbewegungen. Auch die australischen Politikwissenschaftler Dennis Altman und Jonathan Symons (2018: 102) sprechen davon, dass ,[d]er gemeinsame Nenner aller Gegner*innen von Homosexuellenrechten das Bestreben [ist], eine bestimmte Art von Geschlechterregime aufrecht zu erhalten, in dem es eine klare Unterscheidung zwischen Männern und Frauen gibt, und die Annahme, dass sich Kinder nur in einer heterosexuellen Familie gut entwickeln können." Kern der Argumentation ist häufig weniger die Ablehnung von Homosexualität als solcher, sondern die Abwehr der Infragestellung eines binär-Zweigeschlechtlichen Geschlechterwissens.

Für die Formierung als politisches Projekt, welches langfristig den Sprung zum potenziellen Hegemonieprojekt bewältigen möchte, wird das in sich heterogene, autoritär-regressive und antifeministische Projekt darauf angewiesen sein, geteilte ordnungspolitische Entwürfe zu entwickeln, die neben dem Bereich der sozialen Reproduktion auch weitere Stützungsverhältnisse der gegenwärtigen Herrschaftsordnung und damit den gegenwärtigen Regulationsmodus als solchen betreffen.

Für weitergehende Forschung zum Thema bedeutet dies zunächst, theoretische und methodologische Zugänge zu überdenken. Wie an den diskursiven Strategien des autoritär-regressiven Projektes in unserem Untersuchungszeitraum gezeigt werden konnte, benötigt es einen erweiterten Begriff des Politischen, um zu erfassen, wie Akteursgruppen im zivilgesellschaftlichen Raum um Hegemonie ringen - und um die Frage nach dem Erfolg eines politischen Projektes nicht allein an einem konkreten Gesetzgebungsverfahren zu beurteilen.

Denn es gilt zu berücksichtigen, dass die beständigen Angriffe auf die liberale (Geschlechter-)Demokratie vielfältige, teils auch zunächst unsichtbare Effekte haben. Antifeministische und autoritär-regressive Kampagnen zielen auf eine Beeinflussung der Grenzen des Sagbaren und auf die Zurückdrängung progressiver Anliegen, und dies verstärkt im häufig als vorpolitisch verstandenen Raum der Zivil- 
gesellschaft. Sie ringen hier mit anderen, häufig als kritische Zivilgesellschaft beschriebenen progressiv-liberalen politischen Projekten um Hegemonie. Aktuell ist zu beobachten, wie diese Initiativen zunehmend unter Druck geraten, wenn sie durch antifeministische Attacken verstärkt Rechtfertigungszwängen ausgesetzt sind. Aus hegemonietheoretischer Perspektive betrachtet, arbeiten hier neurechte und christlich-rechte AkteurInnen mit verschiedenen dezentralen politischen Projekten an einer Verschiebung gesellschaftlicher Kräfteverhältnisse auf dem Weg zu einem neuen autoritären Ordnungsentwurf. Dem zu begegnen bleibt Aufgabe einer kritischen Zivilgesellschaft, Hand in Hand mit kritischer Wissenschaft.

\section{LITERATUR}

Altman, Dennis/Symons, Jonathan (2018): Queer Wars. Erfolge und Bedrohungen einer globalen Bewegung, Bonn: Sonderausgabe für die Bundeszentrale für politische Bildung.

Antidiskriminierungsstelle des Bundes (2017): Einstellungen gegenüber Lesben, Schwulen und Bisexuellen in Deutschland. Ergebnisse einer bevölkerungsrepräsentativen Umfrage. [https://www.antidiskriminierungsstelle.de/SharedDocs/ Downloads/DE/publikationen/Umfragen/Handout_Themenjahrumfrage_2017. pdf?_blob=publicationFile\&v $=5$; abgerufen am 14.04.2020].

Becker, Lia/Candeias, Mario/Niggemann, Janek/Steckner, Anne (2013): Gramsci lesen: Einstieg in die Gefängnishefte, Hamburg: Argument Verlag.

Billmann, Lucie (Hg.) (2015): Unheilige Allianz. Das Geflecht von christlichen Fundamentalisten und politisch Rechten am Beispiel des Widerstands gegen den Bildungsplan in Baden-Württemberg. [http://www.rosalux.de/fileadmin/rls uploads/pdfs/Materialien/Materialien8_Unheilige_Allianz.pdf; abgerufen am 07.11.2019].

Birsl, Ursula (2017): „Ähnlichkeiten und Unterschiede, Verflechtungen: die säkulare und religiöse Rechte in Deutschland“, in: Christoph Kopke/Wolfgang Kühnel (Hg.), Demokratie, Freiheit und Sicherheit. Festschrift zum 65. Geburtstag von Hans-Gerd Jaschke, Baden-Baden: Nomos, S. 107-127.

Birsl, Ursula (2018): Christliche Rechte: Ein Phänomen der extremen Rechten? [https://www.der-rechte-rand.de/archive/3117/christliche-rechte-phaenomen/; abgerufen am 28.11.2019].

Birsl, Ursula (2019): „Zukunft der Demokratie“. Festrede zur Eröffnung der Sommerakademie „Demokratie gestalten!“ der Begabtenförderungswerke. [http:// www.boeckler.de/pdf/sommerakademie_2019_vortrag_birsl.pdf; abgerufen am 14.04.2020].

Botsch, Gideon (2017): Die Junge Freiheit - Sprachrohr einer radikal-nationalistischen Opposition. [https://www.bpb.de/politik/extremismus/rechtsextremismus 
/230020/die-junge-freiheit-sprachrohr-einer-radikal-nationalistischen-opposi tion; abgerufen am 07.11.2019].

Botsch, Gideon/Kopke, Christoph (2019): ,Umvolkung‘ und ,Volkstod‘. Zur Kontinuität einer extrem rechten Paranoia, Ulm: Verlag Klemm+Oelschläger.

Brand, Ulrich (2013): „State, Context and Correspondence. Contours of a Historical-Materialist Policy Analysis“, in: Österreichische Zeitschrift für Politikwissenschaft 42 (4), S. 425-442.

Buckel, Sonja/Georgi, Fabian/Kannankulam, John/Wissel, Jens (2014): „Theorie, Methoden und Analysen kritischer Europaforschung“, in: Forschungsgruppe Staatsprojekt Europa (Hg.), Kämpfe um Migrationspolitik: Theorie, Methode und Analysen kritischer Europaforschung, Bielefeld: transcript, S. 15-84.

Datta, Neill (2018): Restoring the Natural Order. The Religious Extremists' Vision to Mobilize European Societies Against Human Rights on Sexuality and Reproduction, Brussels: European Parliamentary Forum for Sexual and Reproductive Rights.

Davidson-Schmich, Louise K. (2017): „Amending Germany’s Life Partnerschip Law: Emerging Attention to Lesbians' Concerns“, in: Louise K. DavidsonSchmich (Hg.), Gender, Intersections, and Institutions. Intersectional Group Building Alliances and gaining Voice in Germany, Ann Arbor: University of Michigan Press, S. 203-236.

Decker, Oliver/Brähler, Elmar (Hg.) (2018): Flucht ins Autoritäre. Rechtsextreme Dynamiken in der Mitte der Gesellschaft, Gießen: Psychosozial-Verlag.

Dernbach, Andrea (2017): Die Akzeptanz in Deutschland ist begrenzt. [http://www. tagesspiegel.de/gesellschaft/queerspiegel/studie-zur-homosexualitaet-die-akzep tanz-in-deutschland-ist-begrenzt/19243590.html; abgerufen am 07.11.2019].

Deutschlandfunk (2014): Christliche Medien. ,Scharnierorgane' für rechtes Gedankengut. Sonja Strube im Gespräch mit Katja Schlesinger und Frank Meyer. [https://www.deutschlandfunkkultur.de/christliche-medien-scharnierorgane-fuer -rechtes-gedankengut.1008.de.html?dram:article_id=304657; abgerufen am 07. 11.2019].

Diskursatlas Antifeminismus (2019): Keimzelle der Nation. [http://www.diskurs atlas.de/index.php?title=Keimzelle_der_Nation; abgerufen am 07.11.2019].

Dück, Julia (2014): „Krise und Geschlecht. Überlegungen zu einem feministischmaterialistischen Krisenverständnis“, in: PROKLA 44 (1), Nr. 174, S. 53-70.

Eagleton, Terry (2000): Ideologie: eine Einführung, Stuttgart: Verlag J.B. Metzler.

Eitel, Hanna (2018): Was heißt autoritärer Sog? [http://www.weiterdenken.de/de/ 2018/10/15/was-heisst-autoritaerer-sog; abgerufen am 07.11.2019].

Forster, Edgar (2006): „Männliche Resouveränisierungen“, in: Feministische Studien 24 (2), S. 193-207.

Fuchs, Christian/Middelhoff, Paul (2019): Das Netzwerk der Neuen Rechten, Reinbek: Rowohlt. 
Gehrmann, Anne (2018): Diskursive Praxis und politische Entscheidungen. Eine diskursanalytische Untersuchung der medialen Debatte im Vorfeld der Bundestagsentscheidung zur ,Ehe für alle‘. Unveröffentlichte Masterarbeit, Marburg.

Gessenharter, Wolfgang (1989): „Die ,Neue Rechte“ als Scharnier zwischen Neokonservatismus und Rechtsextremismus in der Bundesrepublik“, in: Rainer Eisfeld/Ingo Müller (Hg.), Gegen Barbarei: Essays Robert M.W. Kempner zu Ehren, Frankfurt a.M.: Athenäum, S. 424-52.

Gessenharter, Wolfgang (1990): „Die Neue Rechte als Scharnier zwischen Neokonservatismus und Rechtsextremismus", in: Rolf Seeliger (Hg.), Grauzone zwischen Union und der Neuen Rechten: Personen, Institutionen, Identifikationen, München: Seeliger, S. 63-77.

Hark, Sabine/Villa, Paula-Irene (Hg.) (2015): Anti-Genderismus. Geschlecht und Sexualität als Schauplätze aktueller politischer Auseinandersetzungen, Bielefeld: transcript.

Häusler, Alexander (2018): Völkisch-autoritärer Populismus: der Rechtsruck in Deutschland und die AfD, Hamburg: VSA.

Heitmeyer, Wilhelm (2018): Autoritäre Versuchungen, Berlin: Suhrkamp.

Henninger, Annette/Backöfer, Ferdinand/Fritzsche, Christopher/Näser-Lather, Marion (2020): „Krise der Geschlechterverhältnisse oder Krisenrhetorik? Antifeministische Bedrohungsszenarien aus regulationstheoretischer Perspektive“, in: Henninger/Birsl, Antifeminismen. ,Krisen'-Diskurse mit gesellschaftsspaltendem Potential?

Henninger, Annette/Birsl, Ursula (Hg.) (2020): Antifeminismen. ,Krisen'-Diskurse mit gesellschaftsspaltendem Potential?, Bielefeld: transcipt.

Hirsch, Joachim (1995): Der nationale Wettbewerbsstaat. Staat, Demokratie und Politik im globalen Kapitalismus, Berlin: ID-Verlag.

Kellershohn, Helmut (2017): Die Neue Rechte: wo sie herkommt, was sie will, wohin sie geht. [https://www.diss-duisburg.de/2017/07/helmut-kellershohn-dieneue-rechte/; abgerufen am 07.11.2019].

Klenner, Christina/Pfahl, Svenja/Neukirch, Sabine/Weßler-Poßberg, Dagmar (2011): „Prekarisierung im Lebenszusammenhang - Bewegung in den Geschlechterarrangements?", in: WSI-Mitteilungen 64 (8), S. 416-422.

Kohlmorgen, Lars (2004): Regulation, Klasse, Geschlecht. Die Konstituierung der Sozialstruktur im Fordismus und Postfordismus, Münster: Westfälisches Dampfboot.

Kováts, Eszter/Põim, Maari (Hg.) (2015): Gender as Symbolic Glue. The Position and Role of Conservative and Far Right Parties in the Anti-Gender Mobilizations in Europe, Berlin: Friedrich-Ebert-Stiftung. [https://library.fes.de/pdf-files/ bueros/budapest/11382.pdf; abgerufen am 07.11.2019].

Kram, Johannes (2018): Ich hab ja nichts gegen Schwule, aber ...: Die schrecklich nette Homophobie in der Mitte der Gesellschaft, Berlin: Querverlag. 
Krüger-Kirn, Helga/Tichy, Leila Zoë (2020): „Elternschaft und Gender Trouble. Inszenierungen moderner und tradierter Mutterbilder", in: Henninger/Birsl, Antifeminismen. ,Krisen“-Diskurse mit gesellschaftsspaltendem Potential?

Kuhar, Roman/Paternotte, David (Hg.) (2017): Anti-Gender Campaigns in Europe: Mobilizing Against Equality, London/New York: Rowman \& Littlefield.

Lang, Juliane (2019): Geschlecht als Kampfarena. Autoritäre Entwicklungen im Bereich von Geschlechter- und Familienpolitiken. [www.gwi-boell.de/de/2019/ 07/03/geschlecht-als-kampfarena; abgerufen am 07.11.2019].

Lang, Juliane/Fritzsche, Christopher (2018): „Backlash, neoreaktionäre Politiken oder Antifeminismus? Forschende Perspektiven auf aktuelle Debatten um Geschlecht", in: Feministische Studien 36 (2), S. 335-46.

Lang, Juliane/Peters, Ulrich (Hg.) (2018): Antifeminismus in Bewegung. Aktuelle Debatten um Geschlecht und sexuelle Vielfalt, Hamburg: Marta-Press.

Langebach, Martin/Raabe, Jan (2016): „Die ,Neue Rechte“ in der Bundesrepublik Deutschland“, in: Virchow/Langebach/Häusler, Handbuch Rechtsextremismus, S. 561-592.

Lenz, Ilse (2013): „Zum Wandel der Geschlechterordnungen im globalisierten flexibilisierten Kapitalismus. Neue Herausforderungen für die Geschlechterforschung“, in: Feministische Studien 31 (1), S. 124-130.

Lenz, Ilse (2018): „Von der Sorgearbeit bis \#metoo. Aktuelle feministische Themen und Debatten in Deutschland“, in: Aus Politik und Zeitschichte 68 (17): S. 2027.

Linde, Désirée (2014): Das Meinungs-Imperium der AfD. [https://www.handels blatt.com/politik/deutschland/beatrix-von-storch-das-meinungs-imperium-derafd/11006550.html?ticket=ST-25981-ZCZ4AMFtz5an6hMsPxHa-ap3; abgerufen am 07.11.2019].

Lipietz, Alain (1985): „Akkumulation, Krisen und Auswege aus der Krise. Einige methodische Überlegungen zum Begriff der ,Regulation“", in: PROKLA 58, S. 109-137.

Ludwig, Gundula (2011): Geschlecht regieren zum Verhältnis von Staat, Subjekt und heteronormativer Hegemonie, Frankfurt a.M./New York: Campus-Verlag.

Marktforschung (2019): Die 20 erfolgreichsten Bloggerinnen. [https://www.markt forschung.de/aktuelles/marktforschung/die-20-erfolgreichsten-bloggerinnen/; abgerufen am 07.11.2019].

Mayer, Stefanie/Sori, Iztok/Sauer, Birgit/Ajanovic, Edma (2018): „Mann, Frau, Volk, Familienidylle, Heteronormativität und Femonationalismus im europäischen rechten Populismus“, in: Feministische Studien 36 (2), S. 269-285.

Meißner, Michael (2017): „Raumordnungspolitik als Verdichtung politischer Kräfteverhältnisse“, in: sublurban zeitschrift für kritische stadtforschung 50 (1/2), S. 21-40.

Meuser, Michael/Nagel, Ulrike (2005): „ExpertInneninterviews - vielfach erprobt, wenig bedacht", in: Alexander Bogner/Beate Littig/Wolfgang Menz (Hg.), Das 
Experteninterview. Theorie, Methode, Anwendung. 2.Aufl., Wiesbaden: VS, S. 71-93.

Nachtwey, Oliver (2016): Die Abstiegsgesellschaft: über das Aufbegehren in der regressiven Moderne, Berlin: Suhrkamp.

Näser-Lather, Marion (2020): „Wissenschaftler_innen vs. Gender Studies. Argumentationen, Wirkungen und Kontexte einer ,wissenschafts'-politischen Debatte“, in: Henninger/Birsl, Antifeminismen. ,Krisen“-Diskurse mit gesellschaftsspaltendem Potential?

Notz, Gisela (2015): Kritik des Familismus: Theorie und soziale Realität eines ideologischen Gemäldes, Stuttgart: Schmetterling Verlag.

Paternotte, David (2018): „Unpacking Oppositional Success: The French Laboratory“, in: Mieke Verloo (Hg.), Varieties of Opposition to Gender Equality in Europe, New York: Routledge, S. 154-171.

Poulantzas, Nicos (2002): Staatstheorie. Politischer Überbau, Ideologie, autoritärer Etatismus, Hamburg: VSA.

Priester, Karin (2016): „Rechtspopulismus - ein umstrittenes theoretisches und politisches Phänomen“, in: Virchow/Langebach/Häusler, Handbuch Rechtsextremismus, S. 533-560.

Reinfeldt, Sebastian (2014): Rechtspopulistische Anschlüsse an den herrschenden Diskurs. [https://www.zeitschrift-luxemburg.de/europa-sprechen/; abgerufen am 07.11.2019].

Reisigl, Martin/Wodak, Ruth (2001): Discourse and Discrimination: Rhetorics of Racism and Antisemitism, London: Routledge.

Sanders, Eike/Jentsch, Ulli/Hansen, Felix (2014): ,Deutschland treibt sich ab`. Organisierter ,Lebensschutz“, christlicher Fundamentalismus, Antifeminismus, Münster: Unrast Verlag.

Sauer, Birgit (2001): Die Asche des Souveräns: Staat und Demokratie in der Geschlechterdebatte, Frankfurt a.M./New York: Campus-Verlag.

Sauer, Birgit (2017): „Gesellschaftstheoretische Überlegungen zum europäischen Rechtspopulismus. Zum Erklärungspotential der Kategorie Geschlecht“, in: Politische Vierteljahresschrift 58 (1), S.1-20.

Scheele, Sebastian (2016): Vom Antifeminismus zum ,Anti-Genderismus"? Eine diskursive Verschiebung und ihre Hintergründe. Keynote auf der Tagung, Gegner*innenaufklärung - Informationen und Analysen zu Anti-Feminismus', Berlin, 31.5.2016. [https://www.gwi-boell.de/sites/default/files/uploads/2016/08/ scheele_diskursive_verschiebung_antifeminismus.pdf; abgerufen am 07.11. 2019].

Schmidt, Matthias (2015): Mehrheit der Deutschen für Homo-Ehe. [https://yougov. de/news/2015/05/29/mehrheit-der-deutschen-fur-ehe-fur-alle/; abgerufen am 07. 11.2019]. 
Schmidt, Matthias (2016): Leicht wachsende Mehrheit für Legalisierung der HomoEhe. [https://yougov.de/news/2016/01/28/leicht-wachsende-mehrheit-fur-legali sierung-der-ho/; abgerufen am 14.11.2019].

Schmincke, Imke (2015): „Das Kind als Chiffre politischer Auseinandersetzung am Beispiel neuer konservativer Protestbewegungen in Frankreich und Deutschland“, in: Sabine Hark/Paula Irene Villa (Hg.), Anti-Genderismus: Sexualität und Geschlecht als Schauplätze aktueller politischer Auseinandersetzungen, Bielefeld: transcript, S. 93-108.

Schreiber, Daniel (2018): „Vorwort von Daniel Schreiber: Queeres Deutschland“, in: Dennis Altman/Jonathan Symons, Queer Wars. Erfolge und Bedrohungen einer globalen Bewegung, Bonn: Sonderausgabe für die Bundeszentrale für politische Bildung, S. 7-12.

Schröder, Jens (2019): IVW-Blitz-Analyse Zeitungen: Welt bricht völlig ein, Zeit und Handelsblatt wachsen dank Digital-Lesern. [https://meedia.de/2019/04/18/ ivw-blitz-analyse-zeitungen-welt-bricht-voellig-ein-zeit-und-handelsblatt-wach sen-dank-digital-lesern/; abgerufen am 07.11.2019].

Schutzbach, Franziska (2018): Die Rhetorik der Rechten, Zürich: XanthippeVerlag.

Stambolis-Ruhstorfer, Michael/Tricou, Josselin (2017): „Resisting ,Gender Theory“ in France: A Fulcrum for Religious Action in a Secular Society“, in: Kuhar/Paternotte, Anti-Gender Campaigns in Europe: Mobilizing Against Equality, S. 79-98.

Strube, Angelika (2012): Das rechte Scharnier. [https://www.publik-forum.de/Reli gion-Kirchen/das-rechte-scharnier\#close; abgerufen am 07.11.2019].

Strube, Angelika (2017): „Christliche Unterstützer der AfD. Milieus, Schnittmengen, Allianzen“, in: Stefan Orth/Volker Resing (Hg.), AfD, Pegida und Co.: Angriff auf die Religion?, Freiburg: Herder-Verlag, S. 58-71.

Syrovatka, Felix (2016): Die Reformpolitik Frankreichs in der Krise: Arbeitsmarktund Rentenpolitik vor dem Hintergrund europäischer Krisenbearbeitung, Wiesbaden: Springer VS.

Teidelbaum, Lucius (2018): Die christliche Rechte in Deutschland. Strukturen, Feindbilder, Allianzen, Münster: Unrast.

Virchow, Fabian/Langebach, Martin/Häusler, Alexander (Hg.) (2016): Handbuch Rechtsextremismus, Wiesbaden: Springer VS.

Weiß, Volker (2017): Die autoritäre Revolte. Die Neue Rechte und der Untergang des Abendlandes, Stuttgart: Klett-Cotta.

Wielowiejski, Patrick (2018): „Identitäre Schwule und bedrohliche Queers. Zum Verhältnis von Homonationalismus und Anti-/G/enderismus im Nationalkonservatismus“, in: Feministische Studien 36 (2), S. 347-356.

Wodak, Ruth/Köhler, Katharina (2010): ,Wer oder was ist ,fremd“? Diskurshistorische Analyse fremdenfeindlicher Rhetorik in Österreich“, in: SWS-Rundschau 50 (1): S. 33-55. 
Wolf, Sebastian (2016): ,Trading out of Crisis‘? Zur Bedeutung von Handelspolitik im europäischen Krisenmanagement. [DOI: http://dx.doi.org/10.15496/publi kation-18315].

\section{QUELLEN}

\section{Textkorpus Diskursanalyse}

Bauer, Jörgen (2013): „Homo-Splitting. Wer schützt die Verfassung vor dem Bundesverfassungsgericht?", in: Charismatismus vom 10.06.2013.

Baschang, Klaus (2011): „,Homo-Ehe“ im protestantischen Pfarrhaus“, in: kath.net vom 03.03.2011.

Bischof Fürst (2015): „Ich lehne die ,Homo-Ehe“, die ,Ehe für alle“ ab“, Nachdruck in: kath.net am 22.06.2015.

Cornides, Jakob (2013): „Homo-Adoption: Weiß die österreichische Regierung, was sie da tut?", in: kath.net vom 27.05.2013.

Fischer, Bärbel (2015a): „Homo-Ehe - ein Menschenrecht?“, in: Freie Welt vom 10.06.2015.

Fischer, Bärbel (2015b): „Beschädigung der EHE ist vorprogrammiert“, in: Freie Welt vom 16.10.2015.

Geis, Norbert (2017): „,Ehe für alle‘. Im Grunde verfassungswidrig“, in: Junge Freiheit 28/17 vom 07.07.2017.

Von Gersdorff, Matthias (2017): „Bundestagsabgeordnete kontaktieren: HomoEhe? - Nein, danke!“, in: Charismatismus vom 29.06.2017.

Heber, Claudia (2017): „Die CDU-Grundfeste sind im Markenkern erschüttert“, in: kath.net vom 28.6.2017.

Hinz, Thorsten (2015): „Erstmal mundtot machen“, in: Junge Freiheit 25/15 vom 12.06.2015.

Krause, Klaus-Peter (2015): „Ehe muss bleiben, was sie immer war“, in: Freie Welt vom 12.06.2015.

Küble, Felizitas (2013): „TV-Kontroverse bei ,Anne Will‘ zum Karlsruher Urteil und zum Adoptionsrecht für Homo-Partnerschaften“, in: Charismatismus vom 13.06.2013.

Küble, Felizitas (2017): „Regensburg: Erklärung von Bischof Dr. Rudolf Voderholzer gegen die ,Homo-Ehe““, Nachdruck in: Charismatismus vom 30.06.2017.

Kuby, Gabriele (2013): „Die Zukunft gehört uns“, in: kath.net vom 18.01.2013.

Kutschera, Ulrich (2017a): „Ehe für alle und der Stiefvater-Effekt“, in: kath.net vom 20.07.2017. [http://www.kath.net/news/60339; abgerufen am 07.11.2019].

Kutschera, Ulrich (2017b): „Homoehe im Lichte der Biologie“, Nachdruck des Interviews des polnischen Wochenmagazins ,Sieci‘ in: kath.net vom 18.09.2017. 
Lombard, Andreas (2013): „Kultur war gestern. Heute opfert der Westen seine Kinder", in: Freie Welt vom 02.07.2013.

Lombard, Andreas (2015): ,,Homosexualität‘ ist ein großangelegtes Täuschungsmanöver", Interview in: Freie Welt vom 03.12.2015.

Müller, Friedrich-Thorsten (2012): „Ein Papa, eine Mama, ganz einfach!“, in: Junge Freiheit 48/12 vom 23.11.2012.

Paulwitz, Michael (2013): „Die CDU sieht rosa“, in: Junge Freiheit 10/13 vom 01.03.2013.

Schwarz, Moritz (2011): „,Klima der Angst““, Interview mit Matthias von Gersdorff in: Junge Freiheit 03/11 vom 14.01.2011.

Spieker, Manfred (2015): „Auf eigene Kinder ausgerichtet“, in: Junge Freiheit 31_32/15 vom 24.07.2015.

Stein, Dieter (2013): „Widerstand ist machbar, Herr Nachbar“, in: Junge Freiheit 04/13 vom 18.01.2013.

Vergely, Bertrand (2013): „Die Diktatur der Konfusion“, Übersetzung des französischen Originals durch Andreas Lombard in: Freie Welt vom 23.04.2013.

Weißmann, Karlheinz (2015): „Die Kontinuität des Lebens sichern“, in: Junge Freiheit 25/15 vom 12.06.2015.

\section{Weitere Quellen}

Paulwitz, Michael (2009): „Die Regenbogenfamilie als Ziel“, in: Junge Freiheit vom 28.08.2009.

\section{Interviews}

Interview I, LSVD, geführt am 02.03.2018.

Interview II, LSVD, geführt am 12.03.2018.

Interview III, Lesbenring, geführt am 11.05.2018.

Interview A, SPD, geführt am 03.04.2019.

Interview B, SPD, geführt am 08.05.2019.

Interview C, Linkspartei, geführt am 06.05.2019.

Interview D, Linkspartei, geführt am 11.06.2019.

Interview E, Grüne, geführt am 06.05.2019.

Interview F, CDU, geführt am 07.06.2019.

Interview G, CDU, geführt am 13.06.2019.

Interview H, CDU, geführt am 12.06.2019.

Interview I, CSU, geführt am 25.07.2019. 
